\title{
European options in a non-linear incomplete market model with default
}

\author{
Miryana Grigorova * $\quad$ Marie-Claire Quenez ${ }^{\dagger}$ Agnès Sulem ${ }^{\ddagger}$
}

February 19, 2019

\begin{abstract}
This paper studies the superhedging prices and the associated superhedging strategies for European options in a non-linear incomplete market model with default. We present the seller's and the buyer's point of view. The underlying market model consists of a risk-free asset and a risky asset driven by a Brownian motion and a compensated default martingale. The portfolio processes follow non-linear dynamics with a non-linear driver $f$. By using a dynamic programming approach, we first provide a dual formulation of the seller's (superhedging) price for the European option as the supremum, over a suitable set of equivalent probability measures $Q \in \mathcal{Q}$, of the $f$ evaluation/expectation under $Q$ of the payoff. We also provide a characterization of the seller's (superhedging) price process as the minimal supersolution of a constrained BSDE with default and a characterization in terms of the minimal weak supersolution of a BSDE with default. By a form of symmetry, we derive corresponding results for the buyer. Our results rely on first establishing a non-linear optional and a non-linear predictable decomposition for processes which are $\mathcal{E}^{f}$-strong supermartingales under $Q$, for all $Q \in \mathcal{Q}$.
\end{abstract}

Key-words: European options, incomplete market, superhedging, non-linear pricing, BSDEs with constraints, $f$-expectation, control problems with non-linear expectation, nonlinear optional decomposition, pricing-hedging duality

\section{Introduction}

We consider a financial market with a default time $\vartheta$. The market contains one risky asset whose price dynamics are driven by a one-dimensional Brownian motion and a compensated

\footnotetext{
*School of Mathematics, University of Leeds, Leeds LS2 9JT, UK, email: miryana_grigorova@yahoo.fr

${ }^{\dagger}$ LPSM, Université Paris 7 Denis Diderot, Boite courrier 7012, 75251 Paris cedex 05, France, email: quenez@lpsm.paris

${ }^{\ddagger}$ Mathrisk, INRIA Paris, 2 rue Simone Iff, CS 42112, 75589 Paris Cedex 12, France, and Université Paris-Est, email: agnes.sulem@inria.fr
} 
default martingale. The market exhibits imperfections which are encoded in the non-linearity of the portfolio dynamics. An important feature of this market is that it is incomplete, in the sense that not every European contingent claim can be replicated by a portfolio. In this framework, we are interested in the problem of pricing and hedging of European options, from the point of view of the seller, and of the buyer.

We recall that in the case of a non-linear complete market model, the seller's (hedging) price of the European option with payoff $\eta$ and maturity $T$ is given by the non-linear $f$ evaluation (expectation) of $\eta$, where $f$ is the non-linear driver of the replicating portfolio (cf. [13] and [17] in the Brownian case, and the recent works [12], [10] and [11] in the default case).

In our framework, since contingent claims are not necessarily replicable, we define the seller's (superhedging) price of the European option at time 0 , denoted by $v_{0}$, as the minimal initial capital which allows him/her to build a (non-linear) portfolio whose terminal value dominates the payoff $\eta$ of the option. We provide a dual formulation of this price as the supremum, over a suitable set of equivalent probability measures $Q \in \mathcal{Q}$, of the $(f, Q)$ evaluation $^{1}$ of the payoff $\eta$, that is,

$$
v_{0}=\sup _{Q \in \mathcal{Q}} \mathcal{E}_{Q, 0, T}^{f}(\eta)
$$

The set $\mathcal{Q}$ is related to the set of the so-called martingale probability measures. In the case when $f$ is linear, our result reduces to the well-known dual representation from the literature on linear incomplete markets (cf. [16] and [18]). We characterize the (superhedging) price process of the European option for the seller in terms of the minimal weak supersolution of a $B S D E$ with default. We also provide another characterization of this price process as the minimal supersolution of a constrained BSDE with default. By a form of symmetry, we derive corresponding results for the buyer's superhedging price.

A crucial step in the proof of these results is to establish a non-linear optional decomposition for processes which are $(f, Q)$-supermartingales for all $Q \in \mathcal{Q}$. This decomposition is the analogue in our framework of the well-known optional decomposition from the linear case (cf. [16] and [18]). We also show that the above non-linear optional decomposition is equivalent to a non-linear predictable decomposition (with constraints).

The paper is organized as follows: in Section 2, we introduce some notation and definitions. In Section 3, we first present our market model (Subsection 3.1); in Subsection 3.2, we define the buyer's and seller's superhedging prices of the European option and we discuss no-arbitrage issues; in Subsection 3.3, we introduce the set $\mathcal{Q}$ of $f$-martingale probability measures and give some properties. In Section 4, we present some of the main results of the paper and in Section 5 we give the proofs of these results. The appendices contain results which are interesting in their own right, besides being useful in the proofs from Section 5 . In Appendix A, we give some useful results on strong $\mathcal{E}$-supermartingale families and processes. Appendix B is devoted to the important non-linear optional and non-linear predictable decompositions. Appendix C contains some complements on BSDEs with a non-positive jump at the default time and Appendix D gathers three useful lemmas.

\footnotetext{
${ }^{1}$ or, in other terms, the $f$-evaluation/expectation under the probability measure $Q$.
} 


\section{Notation and definitions}

Let $(\Omega, \mathcal{G}, P)$ be a complete probability space equipped with two stochastic processes: a unidimensional standard Brownian motion $W$ and a jump process $N$ defined by $N_{t}=\mathbf{1}_{\vartheta \leq t}$ for all $t \in[0, T]$, where $\vartheta$ is a random variable which models a default time. We assume that this default can appear after any fixed time, that is $P(\vartheta \geq t)>0$ for all $t \geq 0$. We denote by $\mathbb{G}=\left\{\mathcal{G}_{t}, t \geq 0\right\}$ the augmented filtration generated by $W$ and $N$. We denote by $\mathcal{P}$ the predictable $\sigma$-algebra. We suppose that $W$ is a $\mathbb{G}$-Brownian motion. Let $\left(\Lambda_{t}\right)$ be the predictable compensator of the nondecreasing process $\left(N_{t}\right)$. Note that $\left(\Lambda_{t \wedge \vartheta}\right)$ is then the predictable compensator of $\left(N_{t \wedge \vartheta}\right)=\left(N_{t}\right)$. By uniqueness of the predictable compensator, $\Lambda_{t \wedge \vartheta}=\Lambda_{t}, t \geq 0$ a.s. We assume that $\Lambda$ is absolutely continuous w.r.t. Lebesgue's measure, so that there exists a nonnegative process $\lambda$, called the intensity process, such that $\Lambda_{t}=$ $\int_{0}^{t} \lambda_{s} d s, t \geq 0$. To simplify the presentation, we suppose that $\lambda$ is bounded. Since $\Lambda_{t \wedge \vartheta}=\Lambda_{t}$, $\lambda$ vanishes after $\vartheta$. Let $M$ be the compensated martingale given by

$$
M_{t}:=N_{t}-\int_{0}^{t} \lambda_{s} d s .
$$

Let $T>0$ be the terminal time. We define the following sets:

- $S^{2}$ is the set of $\mathbb{G}$-adapted RCLL processes $\varphi$ such that $\mathbb{E}\left[\sup _{0 \leq t \leq T}\left|\varphi_{t}\right|^{2}\right]<+\infty$.

- $\mathcal{A}^{2}$ is the set of real-valued non decreasing RCLL $\mathbb{G}$-predictable processes $A$ with $A_{0}=0$ and $\mathbb{E}\left(A_{T}^{2}\right)<\infty$.

- $\mathbb{H}^{2}$ is the set of $\mathbb{G}$-predictable processes $Z$ such that $\|Z\|^{2}:=\mathbb{E}\left[\int_{0}^{T}\left|Z_{t}\right|^{2} d t\right]<\infty$.

- $\mathbb{H}_{\lambda}^{2}:=L^{2}\left(\Omega \times[0, T], \mathcal{P}, \lambda_{t} d P \otimes d t\right)$, equipped with the norm $\|U\|_{\lambda}^{2}:=\mathbb{E}\left[\int_{0}^{T}\left|U_{t}\right|^{2} \lambda_{t} d t\right]<$ $\infty$.

Note that, without loss of generality, we may assume that if $U \in \mathbb{H}_{\lambda}^{2}$, it vanishes after $\vartheta$.

- We denote by $\mathcal{T}_{0}$ the set of stopping times $\tau$ such that $\tau \in[0, T]$ a.s.

- For $S$ in $\mathcal{T}_{0}$, we denote by $\mathcal{T}_{S}$ the set of stopping times $\tau$ such that $S \leq \tau \leq T$ a.s.

As in [21], the notation $\mathbb{S}^{2}$ stands for the vector space of $\mathbb{R}$-valued optional (not necessarily cadlag) processes $\phi$ such that $\|\phi\|_{\mathbb{S}^{2}}^{2}:=E\left[\right.$ ess $\left.\sup _{\tau \in \mathcal{T}_{0}}\left|\phi_{\tau}\right|^{2}\right]<\infty$. By Proposition 2.1 in [21], the space $\mathbb{S}^{2}$ endowed with the norm $\|\cdot \cdot\|_{\mathbb{S}^{2}}$ is a Banach space. We note that the space $S^{2}$ is the sub-space of RCLL processes of $\mathbb{S}^{2}$.

Recall that in this setup, we have a martingale representation theorem with respect to $W$ and $M$ (see [25], [30]).

We give the definition of a $\lambda$-admissible driver: 
Definition 2.1 (Driver, $\lambda$-admissible driver). A function $g$ is said to be a driver if $g: \Omega \times[0, T] \times \mathbb{R}^{3} \rightarrow \mathbb{R} ;(\omega, t, y, z, k) \mapsto g(\omega, t, y, z, k)$ is $\mathcal{P} \otimes \mathcal{B}\left(\mathbb{R}^{3}\right)$ - measurable, and such that $g(., 0,0,0) \in \mathbb{H}^{2}$. A driver $g$ is called a $\lambda$-admissible driver if moreover there exists a constant $C \geq 0$ such that $d P \otimes d t$-a.s. , for each $\left(y_{1}, z_{1}, k_{1}\right),\left(y_{2}, z_{2}, k_{2}\right)$,

$$
\left|g\left(t, y_{1}, z_{1}, k_{1}\right)-g\left(t, y_{2}, z_{2}, k_{2}\right)\right| \leq C\left(\left|y_{1}-y_{2}\right|+\left|z_{1}-z_{2}\right|+\sqrt{\lambda_{t}}\left|k_{1}-k_{2}\right|\right) .
$$

A nonnegative constant $C$ which satisfies this inequality is called a $\lambda$-constant associated with driver $g$.

By condition (2.1) and since $\lambda_{t}=0$ on $\left.] \vartheta, T\right], g$ does not depend on $k$ on $\left.] \vartheta, T\right]$.

Let $g$ be a $\lambda$-admissible driver. For all $\eta \in L^{2}\left(\mathcal{G}_{T}\right)$, there exists a unique solution $(X(T, \eta), Z(T, \eta), K(T, \eta)$ ) (denoted simply by $(X, Z, K))$ in $S^{2} \times \mathbb{H}^{2} \times \mathbb{H}_{\lambda}^{2}$ of the following BSDE (see [10]):

$$
-d X_{t}=g\left(t, X_{t}, Z_{t}, K_{t}\right) d t-Z_{t} d W_{t}-K_{t} d M_{t} ; \quad X_{T}=\eta .
$$

We call $g$-conditional expectation, denoted by $\mathcal{E}^{g}$, the operator defined for each $T^{\prime} \in[0, T]$ and for each $\eta \in L^{2}\left(\mathcal{G}_{T^{\prime}}\right)$ by $\mathcal{E}_{t, T^{\prime}}^{g}(\eta):=X_{t}\left(T^{\prime}, \eta\right)$ a.s. for all $t \in\left[0, T^{\prime}\right]$.

We introduce the following assumption :

Assumption 2.2. Assume that there exists a bounded map

$$
\gamma: \Omega \times[0, T] \times \mathbb{R}^{4} \rightarrow \mathbb{R} ;\left(\omega, t, y, z, k_{1}, k_{2}\right) \mapsto \gamma_{t}^{y, z, k_{1}, k_{2}}(\omega)
$$

$\mathcal{P} \otimes \mathcal{B}\left(\mathbb{R}^{4}\right)$-measurable and satisfying $d P \otimes d t$-a.s., for all $\left(y, z, k_{1}, k_{2}\right) \in \mathbb{R}^{4}$,

$$
g\left(t, y, z, k_{1}\right)-g\left(t, y, z, k_{2}\right) \geq \gamma_{t}^{y, z, k_{1}, k_{2}}\left(k_{1}-k_{2}\right) \lambda_{t},
$$

and

$$
\gamma_{t}^{y, z, k_{1}, k_{2}}>-1
$$

Assumption 2.2 ensures the strict monotonicity of the operator $\mathcal{E}^{g}$ with respect to terminal condition (see [10, Section 3.3]).

Definition 2.3. Let $Y \in \mathbb{S}^{2}$. The process $\left(Y_{t}\right)$ is said to be a strong $\mathcal{E}^{g}$-supermartingale ${ }^{2}$ (resp. martingale) if $\mathcal{E}_{\sigma, \tau}^{g}\left(Y_{\tau}\right) \leq Y_{\sigma}$ (resp. $=Y_{\sigma}$ ) a.s. on $\sigma \leq \tau$, for all $\sigma, \tau \in \mathcal{T}_{0}$.

Note that, by the flow property of BSDEs, for each $\eta \in L^{2}\left(\mathcal{G}_{T}\right)$, the process $\mathcal{E}_{\cdot, T}^{g}(\eta)$ is an $\mathcal{E}^{g}$-martingale. The converse also holds since, if $\left(Y_{t}\right)$ is an $\mathcal{E}^{g}$-martingale, then $Y_{t}=\mathcal{E}_{t, T}^{g}\left(Y_{T}\right)$ for all $t \in[0, T]$ a.s.

\footnotetext{
${ }^{2}$ In the case where $Y$ is moreover RCLL (that is, $Y \in S^{2}$ ), we often omit the term "strong".
} 


\section{The market model and the superhedging prices}

\subsection{The market model $\mathcal{M}^{f}$}

We now consider a financial market which consists of one risk-free asset, whose price process $S^{0}=\left(S_{t}^{0}\right)_{0 \leq t \leq T}$ satisfies $d S_{t}^{0}=S_{t}^{0} r_{t} d t$, and one risky asset with price process $S$ which admits a discontinuity at time $\vartheta$. Throughout the sequel, we consider that the price process $S=\left(S_{t}\right)_{0 \leq t \leq T}$ evolves according to the equation

$$
d S_{t}=S_{t^{-}}\left(\mu_{t} d t+\sigma_{t} d W_{t}+\beta_{t} d M_{t}\right) .
$$

All the processes $\sigma r, \mu, \beta$ are supposed to be predictable (that is $\mathcal{P}$-measurable), satisfying $\sigma_{t}>0 d P \otimes d t$ a.s. and $\beta_{\vartheta}>-1$ a.s., and such that $\sigma, \lambda, \sigma^{-1}, \beta$ are bounded.

We consider an investor, endowed with an initial wealth equal to $x$, who can invest his/her wealth in the two assets of the market. At each time $t$, the investor chooses the amount $\varphi_{t}$ of wealth invested in the risky asset. A process $\varphi .=\left(\varphi_{t}\right)_{0 \leq t \leq T}$ is called a portfolio strategy if it belongs to $\mathbb{H}^{2}$.

The value of the associated portfolio (also called wealth) at time $t$ is denoted by $V_{t}^{x, \varphi}$ (or simply $\left.V_{t}\right)$.

In the classical linear case, the wealth process satisfies the self financing condition:

$$
d V_{t}=\left(r_{t} V_{t}+\varphi_{t}\left(\mu_{t}-r_{t}\right)\right) d t+\varphi_{t} \sigma_{t} d W_{t}+\varphi_{t} \beta_{t} d M_{t} .
$$

Setting $Z_{t}:=\varphi_{t} \sigma_{t}$, we get

$$
d V_{t}=\left(r_{t} V_{t}+Z_{t} \theta_{t}\right) d t+Z_{t} d W_{t}+Z_{t} \sigma_{t}^{-1} \beta_{t} d M_{t},
$$

where $\theta_{t}:=\frac{\mu_{t}-r_{t}}{\sigma_{t}}$.

We assume now that the dynamics of the wealth is nonlinear. More precisely, let $x \in \mathbb{R}$ be an initial wealth and let $\varphi$ in $\mathbb{H}^{2}$ be a portfolio strategy. We suppose that the associated wealth process $V_{t}^{x, \varphi}$ (or simply $V_{t}$ ) satisfies the following (forward) dynamics:

$$
-d V_{t}=f\left(t, V_{t}, \varphi_{t} \sigma_{t}\right) d t-\varphi_{t} \sigma_{t} d W_{t}-\varphi_{t} \beta_{t} d M_{t},
$$

with $V_{0}=x$, where $f$ is a nonlinear $\lambda$-admissible driver which does not depend on $k$, such that $f(t, 0,0)=0 .{ }^{3}$ In the classical linear case (see (3.3)), we have $f(t, y, z)=-r_{t} y-z \theta_{t}$ (which is a $\lambda$-admissible driver).

We have the following lemma.

Lemma 3.1. For each $x \in \mathbb{R}$ and each $\varphi$ in $\mathbb{H}^{2}$, the associated wealth process $\left(V_{t}^{x, \varphi}\right)$ is an $\mathcal{E}^{f}$-martingale.

\footnotetext{
${ }^{3}$ so that $\mathcal{E}_{\cdot, T^{\prime}}^{f}(0)=0$ for all $T^{\prime} \in[0, T]$.
} 
Proof. Let $x \in \mathbb{R}$ and $\varphi$ in $\mathbb{H}^{2}$ be given. We note that the process $\left(V_{t}^{x, \varphi}, \varphi_{t} \sigma_{t}, \varphi_{t} \beta_{t}\right)$ is the solution of the BSDE with default jump associated with driver $f$ and the terminal condition $\eta:=V_{T}^{x, \varphi}$. The result then follows from the flow property of BSDEs.

Remark 3.2. We note that for an arbitrary random variable $\eta \in L^{2}$, there does not necessarily exist a pair of processes $(X, \varphi)$ such that $\left(X_{t}, \varphi_{t} \sigma_{t}, \varphi_{t} \beta_{t}\right)$ is solution of the BSDE with default jump associated with driver $f$ and terminal condition $\eta$, that is, such that $(X, \varphi)$ satisfies the dynamics (3.4) with $X_{T}=\eta$. In other terms, the market is incomplete.

In the sequel, we will often use the following change of variables which maps a process $\varphi \in \mathbb{H}^{2}$ to $Z \in \mathbb{H}^{2}$ defined by $Z_{t}=\varphi_{t} \sigma_{t}$. With this change of variables, the wealth process $V=V_{t}^{x, \varphi}$ (for a given $x \in \mathbb{R}$ ) is the unique process satisfying

$$
-d V_{t}=f\left(t, V_{t}, Z_{t}\right) d t-Z_{t} d W_{t}-Z_{t} \sigma_{t}^{-1} \beta_{t} d M_{t}, V_{0}=x .
$$

In the sequel, our non-linear incomplete market model is denoted by $\mathcal{M}^{f}$.

\subsection{Superhedging prices and no-arbitrage}

Let $\eta$ in $L^{2}\left(\mathcal{G}_{T}\right)$ be the payoff of the European option (with maturity $T$ ). It is called replicable if there exists $x \in \mathbb{R}$ and $\varphi \in \mathbb{H}^{2}$ such that $\eta=V_{T}^{x, \varphi}$ a.s. This is equivalent to the existence of $(X, Z) \in \mathcal{S}^{2} \times \mathbb{H}^{2}$ such that

$$
-d X_{t}=f\left(t, X_{t}, Z_{t}\right) d t-Z_{t} d W_{t}-Z_{t} \sigma_{t}^{-1} \beta_{t} d M_{t}, X_{T}=\eta \text { a.s. }
$$

It is clear that all European contingent claims are not necessarily replicable and so the market is incomplete (cf. also Remark 3.2). We introduce the superhedging price for the seller of the claim with payoff $\eta$ defined as the minimal initial capital which allows the seller to build a superhedging strategy for the claim, that is ${ }^{4}$

$$
v_{0}:=\inf \left\{x \in \mathbb{R}: \exists \varphi \in \mathbb{H}^{2} \text { s.t. } V_{T}^{x, \varphi} \geq \eta \text { a.s. }\right\} .
$$

We introduce the superhedging price for the buyer of the claim with payoff $\eta$ (cf. [29], also [6]) defined as the maximal initial price which allows the buyer to build a superhedging strategy for the claim, that is

$$
\tilde{v}_{0}:=\sup \left\{x \in \mathbb{R}: \exists \varphi \in \mathbb{H}^{2} \text { s.t. } V_{T}^{-x, \varphi}+\eta \geq 0 \text { a.s. }\right\} \text {. }
$$

Note that $\tilde{v}_{0}$ is equal to the opposite of the superhedging price for the seller of the option with payoff $-\eta{ }^{5}$

\footnotetext{
${ }^{4}$ Note that $v_{0} \in \overline{\mathbb{R}}$. We shall see below that, under the assumption that $|\eta|$ is smaller than or equal to the value of a portfolio sufficiently integrable (cf. (4.1)), $v_{0}$ is finite (cf. Theorem 4.1).

${ }^{5}$ Recall that when $\beta=0$ and when the filtration is the natural filtration associated with the Brownian motion, the market is complete and we have $v_{0}=\mathcal{E}_{0, T}^{f}(\eta)$ (cf. [13]) and $\tilde{v}_{0}=-\mathcal{E}_{0, T}^{f}(-\eta)$.
} 
Definition 3.3. Let $x \in \mathbb{R}$. Let $y \in \mathbb{R}$ and $\varphi$ in $\mathbb{H}^{2}$. We say that $(y, \varphi)$ is an arbitrage opportunity for the seller ${ }^{6}$ (resp. for the buyer ${ }^{7}$ ) of the European option with initial price $x$ if

$$
\left.y<x \text { and } V_{T}^{y, \varphi}-\eta \geq 0 \text { a.s. ( resp. } y>x \text { and } V_{T}^{-y, \varphi}+\eta \geq 0\right) \text { a.s. }
$$

Proposition 3.4. Let $x \in \mathbb{R}$. There exists an arbitrage opportunity for the seller (resp. for the buyer) of the European option with price $x$ if and only if $x>v_{0}$ (resp. $x<\tilde{v}_{0}$ )

Proof. The idea of the proof is similar to that of [27]. Suppose that there exists an arbitrage opportunity $(y, \varphi)$ for the seller of the European option with price $x$. Then $y<x$ and $V_{T}^{y, \varphi}-\eta \geq 0$ a.s. Hence, $\varphi$ is a superhedging strategy for the seller associated with the initial wealth $y$. By definition of $v_{0}$ as an infimum (cf. (3.6)), we have $y \geq v_{0}$. Since $x>y$, it follows that $x>v_{0}$. For the converse statement, suppose that $x>v_{0}$. Then, by definition of $v_{0}$ as an infimum, we derive that there exists $y$ with $x>y \geq u_{0}$ and $\varphi \in \mathbb{H}^{2}$ such that $V_{T}^{y, \varphi} \geq \eta$ a.s. Since $y<x$, it follows that $(y, \varphi)$ is an arbitrage opportunity for the seller of the European option with price $x$. The result for the buyer can be shown by using similar arguments.

Definition 3.5. A real number $x$ is called an arbitrage-free price for the European option if there exists no arbitrage opportunity, neither for the seller nor for the buyer.

By Propositions 3.4, we get

Proposition 3.6. If $v_{0}<\tilde{v}_{0}$, there does not exist any arbitrage-free price for the European option. If $v_{0} \geq \tilde{v}_{0}$, the interval $\left[\tilde{v}_{0}, v_{0}\right]$ is the set of all arbitrage-free prices. We call it the arbitrage-free interval for the European option.

\subsection{The set $\mathcal{Q}$ of $f$-martingale probability measures}

As mentioned before, the market $\mathcal{M}^{f}$ is incomplete. We recall that in the linear case, that is, when $f(t, y, z)=-r_{t} y-\theta_{t} z$, a dual representation of $v_{0}$ and $\tilde{v}_{0}$ can be achieved via a martingale approach which is based on the notion of martingale probability measures defined as follows: a probability measure $R$ equivalent to $P$ is called a martingale probability measure if the discounted risky-asset price $\left(e^{-\int_{0}^{t} r_{s} d s} S_{t}\right)$ is a martingale under $R$. It is well known that this is equivalent (still in the linear case) to the following definition given, for example, in [35]: a probability measure $R$ is a martingale probability measure if the discounted (linear) wealth processes are $R$-martingales, that is, for all $x \in \mathbb{R}, \varphi \in \mathbb{H}^{2}$, the process $\left(e^{-\int_{0}^{t} r_{s} d s} \bar{V}_{t}^{x, \varphi}\right)$

\footnotetext{
${ }^{6}$ This means that the seller sells the European option at the price $x$ strictly greater than the amount $y$ which is enough to be hedged (by using the strategy $\varphi$ ). He/she thus makes the profit $x-y>0$ at time 0 .

${ }^{7}$ This means that the buyer buys the European option at the price $x$, stricly smaller than the amount $y$, which, borrowed at time 0 , allows him/her to recover his/her debt at time $T$ (by using the strategy $\varphi$ ). He/she thus makes the profit $y-x>0$ at time 0 .
} 
(where $\bar{V}^{x, \varphi}$ follows the linear dynamics (3.2)) is a martingale under $R$. For example, the probability $R^{0}$ which admits $\zeta_{T}^{0}$ as density with respect to $P$ on $\mathcal{G}_{T}$, where $\left(\zeta_{t}^{0}\right)$ satisfies

$$
d \zeta_{t}^{0}=-\zeta_{t}^{0} \theta_{t} d W_{t} ; \quad \zeta_{0}^{0}=1
$$

is a martingale probability measure. However, there exists more than one martingale probability measure (cf. also the discussion in the sequel (3.13)).

In our non linear framework, by analogy with the linear case, we are thus naturally led to introduce the notion of $\mathcal{E}^{f}$-martingale property under a given probability measure $Q$. To this aim, we first introduce the notion of $f$-evaluation under $Q$.

Let $Q$ be a probability measure, equivalent to $P$. From the $\mathbb{G}$-martingale representation theorem (cf. [30], [26]), its density process $\left(\zeta_{t}\right)$ satisfies

$$
d \zeta_{t}=\zeta_{t^{-}}\left(\alpha_{t} d W_{t}+\nu_{t} d M_{t}\right) ; \zeta_{0}=1
$$

where $\left(\alpha_{t}\right)$ and $\left(\nu_{t}\right)$ are predictable processes with $\nu_{\vartheta \wedge T}>-1$ a.s. By Girsanov's theorem, the process $W_{t}^{Q}:=W_{t}-\int_{0}^{t} \alpha_{s} d s$ is a Brownian motion under $Q$, and the process $M_{t}^{Q}:=$ $M_{t}-\int_{0}^{t} \nu_{s} \lambda_{s} d s$ is a martingale under $Q$.

We define the spaces $S_{Q}^{2}, \mathbb{H}_{Q}^{2}$ and $\mathbb{H}_{Q, \lambda}^{2}$ similarly to $S^{2}, \mathbb{H}^{2}$ and $\mathbb{H}_{\lambda}^{2}$, but under probability $Q$ instead of $P$.

Definition 3.7. We call $f$-evaluation under $Q$, or $(f, Q)$-evaluation in short, denoted by $\mathcal{E}_{Q}^{f}$, the operator defined for each $T^{\prime} \in[0, T]$ and for each $\eta \in L_{Q}^{2}\left(\mathcal{G}_{T^{\prime}}\right)$ by $\mathcal{E}_{Q, t, T^{\prime}}^{f}(\eta):=X_{t}$ for all $t \in\left[0, T^{\prime}\right]$, where $(X, Z, K)$ is the solution in $S_{Q}^{2} \times \mathbb{H}_{Q}^{2} \times \mathbb{H}_{Q, \lambda}^{2}$ of the BSDE under $Q$ associated with driver $f$, terminal time $T^{\prime}$ and terminal condition $\eta$, and driven by $W^{Q}$ and $M^{Q}$, that is ${ }^{8}$

$$
-d X_{t}=f\left(t, X_{t}, Z_{t}\right) d t-Z_{t} d W_{t}^{Q}-K_{t} d M_{t}^{Q} ; \quad X_{T^{\prime}}=\eta .
$$

We note that $\mathcal{E}_{P}^{f}=\mathcal{E}^{f}$.

Definition 3.8. Let $Y \in S_{Q}^{2}$. The process $\left(Y_{t}\right)$ is said to be a (strong) $\mathcal{E}_{Q}^{f}$-martingale, or an $(f, Q)$-martingale, if $\mathcal{E}_{Q, \sigma, \tau}^{f}\left(Y_{\tau}\right)=Y_{\sigma}$ a.s. on $\sigma \leq \tau$, for all $\sigma, \tau \in \mathcal{T}_{0}$.

We now introduce the concept of $f$-martingale probability measure.

Definition 3.9. A probability measure $Q$ equivalent to $P$ is called an $f$-martingale probability measure if for all $x \in \mathbb{R}$ and for all $\varphi \in \mathbb{H}^{2} \cap \mathbb{H}_{Q}^{2}$, the wealth process $V^{x, \varphi}$ is a strong $\mathcal{E}_{Q}^{f}$-martingale, or in other terms an $(f, Q)$-martingale.

We note that $P$ is an $f$-martingale probability measure (cf. Lemma 3.1).

\footnotetext{
${ }^{8}$ We note that since we have a representation theorem for $(Q, \mathbb{G})$-martingales with respect to $W^{Q}$ and $M^{Q}$ (see e.g. Proposition 6 in the appendix of [10]), this BSDE admits a unique solution $(X, Z, K)$ in $S_{Q}^{2} \times \mathbb{H}_{Q}^{2} \times \mathbb{H}_{Q, \lambda}^{2}$.
} 
Remark 3.10. (linear case) Let $R^{0}$ be the martingale probability measure, with density $\zeta^{0}$ defined by (3.9). Suppose $f(t, y, z)=-r_{t} y-\theta_{t} z$. We note that, in this case, the $(f, P)$ martingale property of the (linear) wealth processes (cf. Lemma 3.1) is equivalent to the well-known $R^{0}$-martingale property of the discounted wealth processes. In other terms, the $f$-martingale probability property of $P$ corresponds to the (well-known) martingale probability property of $R^{0}$ (see also Remark 3.14 concerning this correspondance between $P$ and $R^{0}$ ).

Notation: We denote by $\mathcal{Q}$ the set of $f$-martingale probability measures $Q$ such that the coefficients $\left(\alpha_{t}\right)$ and $\left(\nu_{t}\right)$ associated with its density with respect to $P$ (see equation (3.10)) are bounded. We note that $P \in \mathcal{Q}$.

Let $\mathcal{V}$ be the set of bounded predictable processes $\nu$ such that $\nu_{\vartheta \wedge T}>-1$ a.s., which is equivalent to $\nu_{t}>-1$ for all $t \in[0, T] \lambda_{t} d P \otimes d t$-a.s. (cf. Remark 9 in [10]).

Proposition 3.11. (Characterization of $\mathcal{Q}$ ) Let $Q$ be a probability measure equivalent to $P$, such that the coefficients $\alpha$ and $\nu$ of its density (3.10) with respect to $P$ are bounded. The two following assertions are equivalent:

(i) $Q \in \mathcal{Q}$, that is, $Q$ is an $f$-martingale probability measure.

(ii) there exists $\nu \in \mathcal{V}$ such that $Q=Q^{\nu}$, where $Q^{\nu}$ is the probability measure which admits $\zeta_{T}^{\nu}$ as density with respect to $P$ on $\mathcal{G}_{T}$, where $\zeta^{\nu}$ satisfies

$$
d \zeta_{t}^{\nu}=\zeta_{t^{-}}^{\nu}\left(-\nu_{t} \lambda_{t} \beta_{t} \sigma_{t}^{-1} d W_{t}+\nu_{t} d M_{t}\right) ; \zeta_{0}^{\nu}=1
$$

Remark 3.12. We note that the mapping $\nu \mapsto Q^{\nu}$ is a one-to-one mapping that carries $\mathcal{V}$ onto $\mathcal{Q}$. So we have $\mathcal{Q}=\left\{Q^{\nu}, \nu \in \mathcal{V}\right\}$.

For $\nu=0$, we have $Q^{\nu}=Q^{0}=P$. We also note that the set $\mathcal{Q}$ does not depend on the driver $f$.

Proof. Let $Q$ be a probability measure equivalent to $P$, such that the coefficients $\alpha$ and $\nu$ of its density (3.10) with respect to $P$ are bounded. Note that $\left(\nu_{t}\right)$ belongs to $\mathcal{V}$. Let $x \in \mathbb{R}$ and let $\varphi \in \mathbb{H}^{2} \cap \mathbb{H}_{Q}^{2}$. The associated wealth process $V=V^{x, \varphi}$ satisfies (3.5). Expressing $W$ and $M$ in terms of $W^{Q}$ and $M^{Q}$, we get

$$
-d V_{t}^{x, \varphi}=f\left(t, V_{t}^{x, \varphi}, \varphi_{t} \sigma_{t}\right) d t-\varphi_{t} \sigma_{t}\left(\alpha_{t}+\nu_{t} \lambda_{t} \beta_{t} \sigma_{t}^{-1}\right) d t-\varphi_{t} \sigma_{t} d W_{t}^{Q}-\varphi_{t} \beta_{t} d M_{t}^{Q}
$$

Proof. Suppose that $\alpha_{t}=-\nu_{t} \lambda_{t} \beta_{t} \sigma_{t}^{-1} d P \otimes d t$-a.e. Then, the process $\left(V_{t}^{x, \varphi}, \varphi_{t} \sigma_{t}, \varphi_{t} \beta_{t}\right)$ is the solution of the BSDE under $Q$ associated with driver $f$ and terminal condition $V_{T}^{x, \varphi}$. Hence, $V^{x, \varphi}$ is an $\mathcal{E}_{Q}^{f}$-martingale. Conversely, suppose that for all $x \in \mathbb{R}$ and $\varphi \in \mathbb{H}^{2} \cap \mathbb{H}_{Q}^{2}$, the process $V^{x, \varphi}$ is an $\mathcal{E}_{Q}^{f}$-martingale. By Lemma D.1 in the Appendix, the finite variational process $\int_{0}^{\cdot} \varphi_{t} \sigma_{t}\left(\alpha_{t}+\nu_{t} \lambda_{t} \beta_{t} \sigma_{t}^{-1}\right) d t$ is thus equal to 0 , which implies that $\varphi_{t} \sigma_{t}\left(\alpha_{t}+\nu_{t} \lambda_{t} \beta_{t} \sigma_{t}^{-1}\right)=$ $0 d P \otimes d t$-a.e. Since this holds for all $\varphi \in \mathbb{H}^{2} \cap \mathbb{H}_{Q}^{2}$ and since, by assumption, $\sigma_{t}>0$, we derive that $\alpha_{t}=-\nu_{t} \lambda_{t} \beta_{t} \sigma_{t}^{-1} d P \otimes d t$-a.e.

We now provide a connection between $f$-martingale probabilities and martingale probabilities. Let $R$ be a probability measure, equivalent to $P$ such that the coefficients $\alpha$ and 
$\nu$ of its density with respect to $P$ (cf. (3.10)) are bounded. By similar arguments as in the proof of Proposition 3.11, we derive that $R$ is a martingale probability measure if and only if there exists $\nu \in \mathcal{V}$ such that $R=R^{\nu}$, where $R^{\nu}$ is the probability measure with density process $\tilde{\zeta}^{\nu}$ (with respect to $P$ ) satisfying

$$
d \tilde{\zeta}_{t}^{\nu}=\tilde{\zeta}_{t^{-}}^{\nu}\left(\left(-\theta_{t}-\nu_{t} \lambda_{t} \beta_{t} \sigma_{t}^{-1}\right) d W_{t}+\nu_{t} d M_{t}\right) ; \tilde{\zeta}_{0}^{\nu}=1
$$

We denote by $\mathcal{P}$ the set of all such probability measures.

By this observation together with Proposition 3.11, we derive the following result.

Proposition 3.13. There exists a one to one mapping from $\mathcal{Q}$ on $\mathcal{P}$. More precisely, the mapping $T_{\theta}$, which, for each $\nu \in \mathcal{V}$, maps the $f$-martingale probability $Q^{\nu}$ (with density $\zeta^{\nu}$ given by (3.11)) onto the martingale probability measure $R^{\nu}$ (with density $\tilde{\zeta}^{\nu}$ ) is a one to one correspondance from $\mathcal{Q}$ on $\mathcal{P}$.

Remark 3.14. Loosely speaking, the mapping $T_{\theta}$ translates the "Brownian coefficient" (of the density) by $-\theta$. We note that $T_{\theta}(P)=R^{0}$ (which completes the observation made in Remark 3.10 on $P$ and $R^{0}$ ).

\section{Main results}

We now consider a European option with maturity $T$ and payoff $\eta \in L^{2}\left(\mathcal{G}_{T}\right)$ such that there exist $x \in \mathbb{R}$ and $\psi \in \mathbb{H}^{2}$ satisfying

$$
|\eta| \leq V_{T}^{x, \psi}=x-\int_{0}^{T} f\left(s, V_{s}^{x, \psi}, \sigma_{s} \psi_{s}\right) d s+\int_{0}^{T} \psi_{s} \sigma_{s} d W_{s}+\int_{0}^{T} \beta_{s} \psi_{s} d M_{s} \quad \text { a.s. }
$$

One of the main results of this paper is the following pricing-hedging duality formula.

Theorem 4.1 (Pricing-hedging duality). Let $\eta$ be a $\mathcal{G}_{T}$-random variable satisfying Assumption (4.1) with $\psi \in \cap_{\nu \in \mathcal{V}} \mathbb{H}_{Q^{\nu}}^{2}$. The superhedging price for the seller $v_{0}$ of the European option with payoff $\eta$ and maturity $T$ satisfies the equality

$$
v_{0}=\sup _{\nu \in \mathcal{V}} \mathcal{E}_{Q^{\nu}, 0, T}^{f}(\eta)
$$

Remark 4.2. Note that, under the assumption $\psi \in \cap_{\nu \in \mathcal{V}} \mathbb{H}_{Q^{\nu}}^{2}$, we have: for all $\nu \in \mathcal{V}$, the wealth process $V^{x, \psi}$ is an $\mathcal{E}_{Q^{\nu}}^{f}$-martingale since $\left(V^{x, \psi}, \sigma \psi, \beta \psi\right)$ is the solution of the BSDE under $Q^{\nu}$ (driven by $W^{Q^{\nu}}$ and $M^{Q^{\nu}}$ ) associated with driver $f$, terminal time $T$ and terminal condition $V_{T}^{x, \psi}$ (cf. the proof of Proposition 3.11). Now, by Assumption (4.1), we have $|\eta| \leq V_{T}^{x, \psi}$ a.s. It follows that for all $\nu \in \mathcal{V}, \mathcal{E}_{Q^{\nu}, 0, T}^{f}(|\eta|) \leq \mathcal{E}_{Q^{\nu}, 0, T}^{f}\left(V_{T}^{x, \psi}\right)=x$.

For each $\nu \in \mathcal{V}$, the $\left(f, Q^{\nu}\right)$-evaluation can be seen as a nonlinear pricing system:

$$
\mathcal{E}_{Q^{\nu}}^{f}: \eta \mapsto \mathcal{E}_{Q^{\nu}, \cdot, T}^{f}(\eta)
$$

which, to each European option with maturity $T$ and payoff $\eta \in L_{Q^{\nu}}^{2}\left(\mathcal{G}_{T}\right)$, associates the price $\mathcal{E}_{Q^{\nu}, 0, T}^{f}(\eta)$ 
Remark 4.3. (linear case) When $f(t, y, z)=-r_{t} y-\theta_{t} z$, we have for each $\nu$ in $\mathcal{V}, \mathcal{E}_{Q^{\nu}, 0, T}^{f}(\eta)=$ $E_{R^{\nu}}\left(e^{-\int_{0}^{T} r_{s} d s} \eta\right)$. In this case, the operator $\mathcal{E}_{Q^{\nu}}^{f}$ thus reduces to the linear price system associated by duality with the martingale probability measure $Q^{\nu}$ (for more details see [24], [16] section 1.7). Hence, Theorem 4.1 reduces to the well-known dual representation of the superhedging price for the seller of the European option in an incomplete (linear) market (cf. [16] for the case when $\beta=0$ ).

For the proof of the above pricing-hedging duality formula, we refer to Section 5.

Since the superhedging price of the option for the buyer $\tilde{v}_{0}$ is equal to the opposite of the superhedging price for the seller of the option with payoff $-\eta$, we derive from Theorem 4.1 the following dual representation result for $\tilde{v}_{0}$ :

$$
\tilde{v}_{0}=-\sup _{\nu \in \mathcal{V}} \mathcal{E}_{Q^{\nu}, 0, T}^{f}(-\eta) .
$$

Remark 4.4. Note that it is possible that $v_{0}<\tilde{v}_{0}$, and hence, that there does not exist an arbitrage-free price for the European option with payoff $\eta$. A simple example is given by $f(t, y, z)=-|y|$ and $\eta=1$. In this case, we have $v_{0}=e^{-T}$ and $\tilde{v}_{0}=e^{T}$.

Remark 4.5. If $f(t, y, z) \geq-f(t,-y,-z)$ (which is satisfied for example when $f$ is convex with respect to $(y, z))$ then, for all $\nu \in \mathcal{V}$, we have $\mathcal{E}_{Q^{\nu}, 0, T}^{f}(\eta) \geq-\mathcal{E}_{Q^{\nu}, 0, T}^{f}(-\eta)$. By taking the supremum over $\nu \in \mathcal{V}$, using the above dual representations of $v_{0}$ and $\tilde{v}_{0}$, we get $v_{0} \geq \tilde{v}_{0}$.

Moreover, if $f(t, y, z)=-f(t,-y,-z)$ (which is satisfied for example when $f$ is linear), then $v_{0}=\tilde{v}_{0}$, and this constant is the unique arbitrage-free price for the European option with payoff $\eta$.

Theorem 4.1 is based on another important result of the present paper, which extends the well-known optional decomposition theorem from the linear case to the non-linear case. More precisely, we show that every RCLL process which is a (strong) $\mathcal{E}^{f}$-supermartingale under all $f$-martingale probability measures admits an optional $\mathcal{E}^{f}$-decomposition, as stated in the following theorem.

Theorem 4.6 (Optional $\mathcal{E}^{f}$-decomposition). Let $\left(Y_{t}\right)$ be an $R C L L$ optional process belonging to $\cap_{\nu \in \mathcal{V}} S_{Q^{\nu}}^{2}$. Suppose that it is a strong $\left(f, Q^{\nu}\right)$-supermartingale for each $\nu \in \mathcal{V}$. Then, there exists a unique $Z \in \mathbb{H}^{2}$, and a unique nondecreasing optional $R C L L$ process, with $h_{0}=0$ and $E\left(h_{T}^{2}\right)<\infty$ such that

$$
-d Y_{t}=f\left(t, Y_{t}, Z_{t}\right) d t-Z_{t} \sigma_{t}^{-1}\left(\sigma_{t} d W_{t}+\beta_{t} d M_{t}\right)+d h_{t} .
$$

Remark 4.7. In fact we have $Z \in \cap_{\nu \in \mathcal{V}} \mathbb{H}_{Q^{\nu}}^{2}$ and $h \in \cap_{\nu \in \mathcal{V}} S_{Q^{\nu}}^{2}$.

In fact, we prove an even more general decomposition, which holds without the assumption $Y$ RCLL.

Remark 4.8. In the classical linear case when $f$ is given by $f(t, y, z)=-r_{t} y-z \theta_{t}$, the above $\mathcal{E}^{f}$-decomposition corresponds to the well known optional decomposition of an RCLL process, which is a supermartingale under each martingale probability measure, up to a discounting and a change of probability measure (see [16], [18] and [31]). 
We now define the seller's (superhedging) price of the European option at each stopping time $S \in \mathcal{T}_{0}$. To this aim, we first define, for each initial wealth $X \in L^{2}\left(\mathcal{G}_{S}\right)$, a superhedging strategy as a portfolio strategy $\varphi \in \mathbb{H}^{2}$ such that $V_{T}^{S, X, \varphi} \geq \eta$ a.s., where $V^{S, X, \varphi}$ denotes the wealth process associated with initial time $S$ and initial condition $X$. Let $\mathcal{A}_{S}(X)$ be the set of all superhedging strategies associated with initial time $S$ and initial wealth $X$. The seller's (superhedging) price at time $S$ is defined by the random variable

$$
v(S):=\operatorname{essinf}\left\{X \in L^{2}\left(\mathcal{G}_{S}\right), \exists \varphi \in \mathcal{A}_{S}(X)\right\} .
$$

Proposition 4.9. (Seller's superhedging price process) We suppose that Assumption (4.1) holds with $\psi \in \cap_{\nu \in \mathcal{V}} \mathbb{H}_{Q^{\nu}}^{2}$. For each $S \in \mathcal{T}_{0}$, we have

$$
v(S)=e s s \sup _{\nu \in \mathcal{V}_{S}} \mathcal{E}_{Q^{\nu}, S, T}^{f}(\eta) \quad \text { a.s. }
$$

Moreover, there exists a unique process $\left(X_{t}\right) \in S^{2}$ such that for each $S \in \mathcal{T}_{0}, v(S)=X_{S}$ a.s. We call $\left(X_{t}\right)$ the seller's (superhedging) price process of the European option.

Remark 4.10. When there is no default in the market, the filtration $\mathbb{G}$ is the one associated with the Brownian motion $W$, and in the dynamics of the price process $\left(S_{t}\right)$ and of the wealth process $\left(V_{t}\right), M=0$ and $\beta=0$. Hence, the market is complete, and we have $\mathcal{V}=\{0\}$ and $\mathcal{Q}=\{P\}$. We derive that the seller's price process satisfies $X_{t}=\mathcal{E}_{t, T}^{f}(\eta)$ for all $t \in[0, T]$ a.s. By symmetry, the buyer's price process corresponds to the process $-\mathcal{E}_{t, T}^{f}(-\eta), 0 \leq t \leq T$.

We now introduce the notion of weak supersolution of the BSDE with driver $f$ and terminal condition $\eta$.

Definition 4.11. Let $\eta \in L^{2}\left(\mathcal{G}_{T}\right)$. A process $X^{\prime} \in S^{2}$ is said to be a weak supersolution of the BSDE with driver $f$ and terminal condition $\eta$ if there exist $Z^{\prime} \in \mathbb{H}^{2}$, and a nondecreasing optional RCLL process $h^{\prime}$, with $h_{0}^{\prime}=0$ and $E\left[\left(h_{T}^{\prime}\right)^{2}\right]<\infty$ such that

$$
-d X_{t}^{\prime}=f\left(t, X_{t}^{\prime}, Z_{t}^{\prime}\right) d t+d h_{t}^{\prime}-\sigma_{s}^{-1} Z_{s}^{\prime}\left(\sigma_{s} d W_{s}+\beta_{s} d M_{s}\right) ; \quad X_{T}^{\prime}=\eta \text { a.s. }
$$

Remark 4.12. We call the above solution a weak supersolution of the BSDE because the associated non decreasing right-continuous process is optional but not necessarily predictable contrary to classical supersolutions of BSDEs.

We have the following infinitesimal characterization of the seller's superhedging price process:

Theorem 4.13. (Infinitesimal characterization I) Let $\eta$ be a $\mathcal{G}_{T}$-random variable satisfying Assumption (4.1). The seller's superhedging price $\left(X_{t}\right)$ is a weak supersolution of the BSDE with driver $f$ and terminal condition $\eta$ from Definition 4.11, that is, there exists a unique process $Z \in \mathbb{H}^{2}$ and a unique nondecreasing optional $R C L L$ process $h$, with $h_{0}=0$ and $E\left[\left(h_{T}\right)^{2}\right]<\infty$ satisfying the equation (4.4). Moreover, it is the minimal one, that is, if $\left(X_{t}^{\prime}\right)$ is another weak supersolution, then $X_{t}^{\prime} \geq X_{t}$ for all $t \in[0, T]$ a.s.

Furthermore, the portfolio strategy $\varphi^{*}:=\sigma^{-1} Z$ is a superhedging strategy for the seller, that is, $\varphi^{*} \in \mathcal{A}\left(v_{0}\right)$. 
We now introduce the notion of a supersolution of the constrained BSDE with driver $f$ and terminal condition $\eta$.

Definition 4.14. Let $\eta \in L^{2}\left(\mathcal{G}_{T}\right)$. A process $X^{\prime} \in S^{2}$ is said to be a supersolution of the constrained BSDE with driver $f$ and terminal condition $\eta$ if there exists a process $\left(Z^{\prime}, K^{\prime}, A^{\prime}\right) \in \mathbb{H}^{2} \times \mathbb{H}_{\lambda}^{2} \times \mathcal{A}^{2}$ such that

$$
\begin{aligned}
& -d X_{t}^{\prime}=f\left(t, X_{t}^{\prime}, Z_{t}^{\prime}\right) d t+d A_{t}^{\prime}-Z_{t}^{\prime} d W_{t}-K_{t}^{\prime} d M_{t} ; \quad X_{T}^{\prime}=\eta \text { a.s.; } \\
& A^{\prime}+\int_{0}\left(K_{s}^{\prime}-\beta_{s} \sigma_{s}^{-1} Z_{s}^{\prime}\right) \lambda_{s} d s \in \mathcal{A}^{2} \quad \text { and } \quad\left(K_{t}^{\prime}-\beta_{t} \sigma_{t}^{-1} Z_{t}^{\prime}\right) \lambda_{t} \leq 0, t \in[0, T], d P \otimes d t-\text { a.e. }
\end{aligned}
$$

Theorem 4.15. (Infinitesimal characterization II) The seller's superhedging price process $\left(X_{t}\right)$ is a supersolution of the constrained BSDE associated with driver $f$ and terminal condition $\eta$ from Definition 4.14 , that is, there exists a unique process $(Z, K, A) \in \mathbb{H}^{2} \times \mathbb{H}_{\lambda}^{2} \times \mathcal{A}^{2}$ such that $(X, Z, K, A)$ satisfies (4.5) and (4.6). Moreover, it is the minimal one.

Furthermore, the portfolio strategy $\varphi^{*}:=\sigma^{-1} Z$ is a superhedging strategy for the seller, that is, $\varphi^{*} \in \mathcal{A}\left(v_{0}\right)$.

Remark 4.16. Recall that the buyer's superhedging price $\tilde{v}_{0}$ for the European option with payoff $\eta$ is equal to the opposite of the seller's superhedging price for the European option with payoff $-\eta$ (cf. Section 3.2). From this and from the results on the seller's superhedging price, we derive the corresponding results for the buyer's superhedging price.

\section{Proofs of the main results}

In order to prove Theorem 4.1, we will work under the primitive probability $P$, which will allow us to solve the problems under weaker integrability conditions.

To this aim, we introduce a family of drivers $\left(f^{\nu}, \nu \in \mathcal{V}\right)$.

Definition 5.1 (Driver $f^{\nu}$ and $\mathcal{E}^{\nu}$-expectation). For $\nu \in \mathcal{V}$, we define

$$
f^{\nu}(\omega, t, y, z, k):=f(\omega, t, y, z)+\nu_{t}(\omega) \lambda_{t}(\omega)\left(k-\beta_{t}(\omega) \sigma_{t}^{-1}(\omega) z\right) .
$$

The mapping $f^{\nu}$ is a $\lambda$-admissible driver ${ }^{9}$.

The associated non-linear family of operators, denoted by $\mathcal{E}^{f^{\nu}}$ or, simply, $\mathcal{E}^{\nu}$, is defined as follows: for each $T^{\prime} \leq T$ and each $\eta \in L^{2}\left(\mathcal{G}_{T^{\prime}}\right)$, we have $\mathcal{E}_{\cdot, T^{\prime}}^{\nu}(\eta):=X^{\nu}$, where $\left(X^{\nu}, Z^{\nu}, K^{\nu}\right)$ is the unique solution in $S^{2} \times \mathbb{H}^{2} \times \mathbb{H}_{\lambda}^{2}$ of the $B S D E$

$$
-d X_{t}^{\nu}=\left(f\left(t, X_{t}^{\nu}, Z_{t}^{\nu}\right)+\nu_{t} \lambda_{t}\left(K_{t}^{\nu}-\beta_{t} \sigma_{t}^{-1} Z_{t}^{\nu}\right)\right) d t-Z_{t}^{\nu} d W_{t}-K_{t}^{\nu} d M_{t} ; X_{T^{\prime}}^{\nu}=\eta .
$$

\footnotetext{
${ }^{9}$ Since $\nu$ is a predictable process, $f^{\nu}$ is $\mathcal{P} \otimes \mathcal{B}\left(\mathbb{R}^{3}\right)-$ measurable. As, moreover, $\nu$ is bounded, $f^{\nu}$ is a $\lambda$-admissible driver.
} 
Remark 5.2. By Proposition 3.11, for each $\nu \in \mathcal{V}$, for all $T^{\prime} \leq T$ and $\eta \in L^{2}\left(\mathcal{G}_{T^{\prime}}\right) \cap$ $L_{Q^{\nu}}^{2}\left(\mathcal{G}_{T^{\prime}}\right)$, we derive that the $\left(f^{\nu}, P\right)$-evaluation of $\eta$ is equal to its $\left(f, Q^{\nu}\right)$-evaluation, that is,

$$
\mathcal{E}_{\cdot, T^{\prime}}^{\nu}(\eta)=\mathcal{E}_{Q^{\nu},, T^{\prime}}^{f}(\eta) .
$$

We place ourselves under the assumption (4.1).

For each $S \in \mathcal{T}$, we define the $\mathcal{F}_{S}$-measurable random variable:

$$
X(S):=e s s \sup _{\nu \in \mathcal{V}_{S}} \mathcal{E}_{S, T}^{\nu}(\eta)
$$

Let us recall the definition of an admissible family of random variables indexed by stopping times in $\mathcal{T}_{0}$ (or $\mathcal{T}_{0}$-system in the vocabulary of Dellacherie and Lenglart [8]).

Definition 5.3. We say that a family $Y=(Y(S), S \in \mathcal{T})$ is admissible if it satisfies the following conditions

1. For all $S \in \mathcal{T}, Y(S)$ is a real-valued $\mathcal{G}_{S}$-measurable random variable.

2. For all $S, S^{\prime} \in \mathcal{T}, Y(S)=Y\left(S^{\prime}\right)$ a.s. on $\left\{S=S^{\prime}\right\}$.

Moreover, we say that an admissible family $Y$ is uniformly square-integrable if $\mathbb{E}\left[\right.$ ess $\left.\sup _{S \in \mathcal{T}}(Y(S))^{2}\right]<\infty$.

Lemma 5.4. The family of random variables $(X(S), S \in \mathcal{T})$ is an admissible family. Moreover, for each $S \in \mathcal{T}$, there exists a sequence of controls $\left(\nu^{n}\right)_{n \in \mathbb{N}}$ with $\nu^{n} \in \mathcal{V}_{S}$ for all $n$, such that the sequence $\left(\mathcal{E}_{S, T}^{\nu^{n}}(\eta)\right)_{n \in \mathbb{N}}$ is nondecreasing and satisfies:

$$
X(S)=\lim _{n \rightarrow \infty} \mathcal{E}_{S, T}^{\nu^{n}}(\eta) \text { a.s. }
$$

Proof. By definition (5.2), for each $S \in \mathcal{T}, X(S)$ is $\mathcal{G}_{S}$-measurable as the essential supremum of $\mathcal{G}_{S^{-}}$-measurable random variables. Let $S, S^{\prime} \in \mathcal{T}$ such that $S=S^{\prime}$ a.s. We have $\mathcal{E}_{S, T}^{\nu}(\eta)=$ $\mathcal{E}_{S^{\prime}, T}^{\nu}(\eta)$ a.s. for all $\nu \in \mathcal{V}$. Hence, ess $\sup _{\nu \in \mathcal{V}} \mathcal{E}_{S, T}^{\nu}(\eta)=e s s \sup _{\nu \in \mathcal{V}} \mathcal{E}_{S^{\prime}, T}^{\nu}(\eta)$ a.s. From this, together with (5.2), we get $X(S)=X\left(S^{\prime}\right)$ a.s. The admissibility of the value family is thus proven.

Let us show the second assertion. By a classical result on essential suprema, it is sufficient to prove that, for each $S \in \mathcal{T}$, the set $\left\{\mathcal{E}_{S, T}^{\nu}(\eta), \nu \in \mathcal{V}_{S}\right\}$ is stable under pairwise maximization. Indeed, let $\nu, \nu^{\prime} \in \mathcal{V}_{S}$. Set $A:=\left\{\mathcal{E}_{S, T}^{\nu^{\prime}}(\eta) \leq \mathcal{E}_{S, T}^{\nu}(\eta)\right\}$. We have $A \in \mathcal{F}_{S}$. Set $\tilde{\nu}:=\nu \mathbf{1}_{A}+\nu^{\prime} \mathbf{1}_{A^{c}}$. Then $\tilde{\nu} \in \mathcal{V}_{S}$. We have $\mathcal{E}_{S, T}^{\tilde{\nu}}(\eta) \mathbf{1}_{A}=\mathcal{E}_{S, T}^{\tilde{\nu}^{\tilde{1}} \mathbf{1}_{A}}\left(\eta \mathbf{1}_{A}\right)=\mathcal{E}_{S, T}^{f^{\nu} \mathbf{1}_{A}}\left(\eta \mathbf{1}_{A}\right)=\mathcal{E}_{S, T}^{\nu}(\eta) \mathbf{1}_{A}$ a.s. and similarly on $A^{c}$. It follows that $\mathcal{E}_{S, T}^{\nu}(\eta)=\mathcal{E}_{S, T}^{\nu}(\eta) \mathbf{1}_{A}+\mathcal{E}_{S, T}^{\nu^{\prime}}(\eta) \mathbf{1}_{A^{c}}=\mathcal{E}_{S, T}^{\nu}(\eta) \vee \mathcal{E}_{S, T}^{\nu^{\prime}}(\eta)$ a.s. The proof is thus complete.

Let $g$ be a $\lambda$-admissible driver satisfying Assumption 2.2. We give the definition of an $\mathcal{E}^{g}$-supermartingale (resp. $\mathcal{E}^{g}$ - submartingale, $\mathcal{E}^{g}$-martingale) family. ${ }^{10}$.

\footnotetext{
${ }^{10}$ When $g=0$, it reduces to the notion of supermartingale family, or supermartingale $\mathcal{T}$-system in the terminology of Dellacherie-Lenglart [8]
} 
Definition 5.5. A uniformly square integrable admissible family $(Y(S), S \in \mathcal{T})$ is said to be an $\mathcal{E}^{g}$-supermartingale (resp. $\mathcal{E}^{g}$ - submartingale, $\mathcal{E}^{g}$-martingale) family if for all $S, S^{\prime} \in$ $\mathcal{T}$ such that $S \geq S^{\prime}$ a.s., $\mathcal{E}_{S^{\prime}, S}^{g}(Y(S)) \leq$ (resp. $\geq$, =) $Y\left(S^{\prime}\right)$ a.s.

Lemma 5.6. The family $(X(S), S \in \mathcal{T})$ is the smallest admissible family such that for each $\nu \in \mathcal{V}$, it is an $\mathcal{E}^{\nu}$-supermartingale family ${ }^{11}$ satisfying for all $S \in \mathcal{T}_{0}, X(T)=\eta$ a.s.

Proof. We first note that, by definition of $X(T)$, we have $X(T)=\eta$ a.s.

Fix $S \in \mathcal{T}_{S^{\prime}}$ a.s. There exists an optimizing sequence of controls $\left(\nu^{n}\right)_{n \in \mathbb{N}}$ with $\left(\nu^{n}\right)$ in $\mathcal{V}_{S}$ such that equality (5.3) holds. Let $\nu \in \mathcal{V}$. By the continuity of $\mathcal{E}^{\nu}$, we have $\mathcal{E}_{S^{\prime}, S}^{\nu}(X(S))=\lim _{n \rightarrow \infty} \mathcal{E}_{S^{\prime}, S}^{\nu}\left(\mathcal{E}_{S, T}^{\nu^{n}}(\eta)\right)$ a.s.

We define for each $n$ the control $\tilde{\nu}_{t}^{n}:=\nu_{t} \mathbf{1}_{\left[S^{\prime}, S\right]}(t)+\nu_{t}^{n} \mathbf{1}_{[S, T]}(t)$, which belongs to $\mathcal{V}_{S^{\prime}}$. Notice that $f^{\tilde{\nu}^{n}}=f^{\nu} \mathbf{1}_{\left[S^{\prime}, S\right]}+f^{\nu^{n}} \mathbf{1}_{[S, T]}$, which implies that $\mathcal{E}_{S^{\prime}, S}^{\nu}\left(\mathcal{E}_{S, T}^{\nu^{n}}(\eta)\right)=\mathcal{E}_{S^{\prime}, S}^{\tilde{\mathcal{L}}^{n}}\left(\mathcal{E}_{S, T}^{\tilde{\nu}^{n}}(\eta)\right)=$ $\mathcal{E}_{S^{\prime}, T}^{\tilde{\nu}^{n}}(\eta)$ a.s. Hence, we obtain $\mathcal{E}_{S^{\prime}, S}^{\nu}(X(S))=\lim _{n \rightarrow \infty} \mathcal{E}_{S^{\prime}, T}^{\tilde{\nu}^{n}}(\eta) \leq X\left(S^{\prime}\right)$ a.s., where the last equality follows from the definition of $X\left(S^{\prime}\right)$. We now show the minimality property. Let $\left(X^{\prime}(S), S \in \mathcal{T}\right)$ be an admissible family such that for each $\nu \in \mathcal{V}$, it is an $\mathcal{E}^{\nu}$-supermartingale family satisfying $X^{\prime}(T)=\eta$ a.s. By the properties of $X^{\prime}$, for all $S \in \mathcal{T}$, and all $\nu \in \mathcal{V}$, we have $X^{\prime}(S) \geq \mathcal{E}_{S, T}^{\nu}\left(X^{\prime}(T)\right)=\mathcal{E}_{S, T}^{\nu}(\eta)$ a.s. Taking the essential supremum over $\nu \in \mathcal{V}_{S}$, we deduce $X^{\prime}(S) \geq X(S)$ a.s.

Using the above Lemma, we get the following result.

Proposition 5.7. There exists an $R C L L$ adapted process $\left(X_{t}\right) \in S^{2}$ which aggregates the value family $(X(S))$. The process $\left(X_{t}\right)$ is a strong $\mathcal{E}^{\nu}$-supermartingale for all $\nu \in \mathcal{V}$ and $X_{T}=$ $\eta$ a.s. Moreover, the process $\left(X_{t}\right)$ is the smallest process in $S^{2}$ satisfying these properties.

Proof. Since $\mathcal{V}$ is nonempty (in particular $0 \in \mathcal{V}$ ), Lemma 5.6 implies that the value family $(X(S))$ is a strong $\mathcal{E}^{0}$-supermartingale family. By Lemma A.1 in the Appendix, there exists a r.u.s.c. optional process $\left(X_{t}\right)$ such that $\mathbb{E}\left[\right.$ ess $\left.\sup _{S \in \mathcal{T}} X_{S}^{2}\right]<\infty$ which aggregates the family $(X(S), S \in \mathcal{T})$ with $X_{T}=\eta$ a.s. Moreover, by Lemma 5.6, $\left(X_{t}\right)$ is the minimal optional process which is a strong $\mathcal{E}^{\nu}$-supermartingale for all $\nu \in \mathcal{V}$, with terminal value greater than or equal to $\eta$.

By Proposition A.6 in the Appendix, we derive that $\left(X_{t}\right)$ is a right-continuous (leftlimited) process and belongs to $S^{2}$.

From this lemma together with Theorem B.4 applied to the right-continuous process $\left(X_{t}\right)$, we get the following result.

Lemma 5.8 (Optional $\mathcal{E}^{f}$-decomposition of the value process). There exists a unique $Z \in \mathbb{H}^{2}$ and a unique nondecreasing optional $R C L L$ process $h$, with $h_{0}=0$ and $E\left[h_{T}^{2}\right]<\infty$ such that

$$
X_{t}=X_{0}-\int_{0}^{t} f\left(s, X_{s}, Z_{s}\right) d s+\int_{0}^{t} \sigma_{s}^{-1} Z_{s}\left(\sigma_{s} d W_{s}+\beta_{s} d M_{s}\right)-h_{t}, 0 \leq t \leq T \quad \text { a.s. }
$$

\footnotetext{
${ }^{11}$ that is an $\mathcal{E}^{f^{\nu}}$-supermartingale family
} 
Remark 5.9. Suppose that $\psi \in \cap_{\nu \in \mathcal{V}} \mathbb{H}_{Q^{\nu}}^{2}$ in Assumption (4.1). Then, $Z \in \cap_{\nu \in \mathcal{V}} \mathbb{H}_{Q^{\nu}}^{2}$ and $h \in \cap_{\nu \in \mathcal{V}} S_{Q^{\nu}}^{2}$.

Using this lemma, we provide a dual representation for the seller's superhedging price $v_{0}$ in terms of the value process $\left(X_{t}\right)$ (at time 0 ).

Theorem 5.10 (Dual representation). The seller's superhedging price $v_{0}$ of the European option is equal to the value function at time 0 (cf. (5.2)) of the non-linear control problem, that is

$$
v_{0}=\sup _{\nu \in \mathcal{V}} \mathcal{E}_{0, T}^{\nu}(\eta)
$$

Moreover, the portfolio strategy $\varphi^{*}:=\sigma^{-1} Z$, where the process $Z$ is the one from the $\mathcal{E}^{f}$ optional decomposition of the value process $X$ from Theorem B.4, is a superhedging strategy for the seller, that is, $V_{T}^{v_{0}, \varphi^{*}} \geq \eta$ a.s.

Proof. For each $x \in \mathbb{R}$, let $\mathcal{A}(x):=\left\{\varphi \in \mathbb{H}^{2}\right.$ s.t. $V_{T}^{x, \varphi} \geq \eta$ a.s. $\}$. Let $\mathcal{H}$ be the set of initial capitals which allow the seller to be "super-hedged", that is $\mathcal{H}=\{x \in \mathbb{R}: \exists \varphi \in \mathcal{A}(x)\}$. From the definition of $v_{0}$ (see (3.6)), we have $v_{0}=\inf \mathcal{H}$. We first show that

$$
v_{0} \geq \sup _{\nu \in \mathcal{V}} \mathcal{E}_{0, T}^{\nu}(\eta)
$$

Let $x \in \mathcal{H}$. There exists $\varphi \in \mathbb{H}^{2}$ such that $V_{T}^{x, \varphi} \geq \eta$ a.s. Let $\nu \in \mathcal{V}$. By taking the $\mathcal{E}^{\nu}$-evaluation in the above inequality, using the monotonicity of $\mathcal{E}^{\nu}$ and the $\mathcal{E}^{\nu}$-martingale property of the wealth process $V^{x, \varphi}$, we obtain $x=\mathcal{E}_{0, T}^{\nu}\left(V_{T}^{x, \varphi}\right) \geq \mathcal{E}_{0, T}^{\nu}(\eta)$. By arbitrariness of $\nu \in \mathcal{V}$, we get $x \geq \sup _{\nu \in \mathcal{V}} \mathcal{E}_{0, T}^{\nu}(\eta)$, which holds for all $x \in \mathcal{H}$. By taking the infimum over $x \in \mathcal{H}$, we derive the desired inequality (5.6). Since, by definition of $X_{0}$, we have $X_{0}=\sup _{\nu \in \mathcal{V}} \mathcal{E}_{0, T}^{\nu}(\eta)$, the inequality (5.6) can be written as $v_{0} \geq X_{0}$.

We now show the converse inequality, that is, $X_{0} \geq v_{0}$. Since $v_{0}=\inf \mathcal{H}$, it is sufficient to show that the portfolio strategy $\varphi^{*}:=\sigma^{-1} Z$ is a superhedging strategy for the seller associated with the initial capital $X_{0}$, that is,

$$
\varphi^{*} \in \mathcal{A}\left(X_{0}\right) .
$$

We consider the portfolio associated with the initial capital $X_{0}$ and the strategy $\varphi^{*}$. By (3.4)-(3.5), the value of this portfolio $\left(V_{t}^{X_{0}, \varphi^{*}}\right)$ satisfies the following forward equation:

$$
V_{t}^{X_{0}, \varphi^{*}}=X_{0}-\int_{0}^{t} f\left(s, V_{s}^{X_{0}, \varphi^{*}}, Z_{s}\right) d s+\int_{0}^{t} \sigma_{s}^{-1} Z_{s}\left(\sigma_{s} d W_{s}+\beta_{s} d M_{s}\right), 0 \leq t \leq T \quad \text { a.s. }
$$

Moreover, by the optional $\mathcal{E}^{f}$-decomposition of the value process $\left(X_{t}\right)$ (cf. Lemma 5.8), the process $\left(X_{t}\right)$ satisfies the forward $\operatorname{SDE}(5.4)$. Now, $\left(h_{t}\right)$ is nondecreasing. Hence, by the comparison result for forward differential equations, we get $V_{T}^{X_{0}, \varphi^{*}} \geq X_{T}$ a.s. Since $X_{T}=\eta$ a.s., we get $V_{T}^{X_{0}, \varphi^{*}} \geq \eta$ a.s., which implies the desired property (5.7). We thus derive that $X_{0} \in \mathcal{H}$, and hence that $X_{0} \geq v_{0}$. Since $X_{0} \leq v_{0}$, we deduce the equality $X_{0}=v_{0}$. Moreover, by (5.7), we derive that $\varphi^{*} \in \mathcal{A}\left(v_{0}\right)$, which completes the proof. 
Remark 5.11. Some related results are given in [2] for European options in a Brownian framework.

From this result, we deduce the pricing-hedging duality formula (in terms of the $f$ martingale probability measures) stated in Theorem 4.1.

Proof of Theorem 4.1 (Pricing-hedging duality): The proof follows from the previous Theorem 5.10 and from Remark 5.2. Indeed, under an additional integrability condition $\psi \in \cap_{\nu \in \mathcal{V}} \mathbb{H}_{Q^{\nu}}^{2}$ on the process $\psi$ from Assumption (4.1), by Remark 5.2, the above dual representation of the superhedging price can be written in terms of the $f$-martingale probability measures, that is

$$
v_{0}=\sup _{\nu \in \mathcal{V}} \mathcal{E}_{Q^{\nu}, 0, T}^{f}(\eta),
$$

which ends the proof of Theorem 4.1.

Proof of Proposition 4.9 (Seller's superhedging price process): Using Lemma 5.8 and similar arguments to those used in the proof of Theorem 5.10, one can show that for each $S \in \mathcal{T}$, we have $v(S)=X_{S}$ a.s., which gives the desired result.

Proof of Theorem 4.13 (Infinitesimal characterization I): The proof relies on Lemma 5.8 and Proposition 5.7. We have $X_{T}=\eta$ a.s. By Lemma 5.8, we derive that the process $X$ is a weak supersolution. It remains to show that it is the minimal one. Let $X^{\prime}$ be another weak supersolution. Hence, there exists $Z^{\prime} \in \mathbb{H}^{2}$ and a nondecreasing optional RCLL process $h^{\prime}$, with $h_{0}^{\prime}=0$ and $E\left[\left(h_{T}^{\prime}\right)^{2}\right]<\infty$ such that (4.4) holds. Let $\nu \in \mathcal{V}$. We show that $X^{\prime}$ is an $\mathcal{E}^{\nu}$-strong supermartingale. Let $\sigma \in \mathcal{T}_{0}$ and let $\tau \in \mathcal{T}_{\sigma}$. We have to show that $\mathcal{E}_{\sigma, \tau}^{\nu}\left(X_{\tau}^{\prime}\right) \leq X_{\sigma}^{\prime}$ a.s. By definition, the process $\mathcal{E}_{,, \tau}^{\nu}\left(X_{\tau}^{\prime}\right)$ is the solution of the BSDE associated with driver $f$, terminal time $\tau$ and terminal condition $X_{\tau}^{\prime}$. Now, the process $\left(X_{t \wedge \tau}^{\prime}\right)$ is the solution of the BSDE associated with generalized (optional) driver $f(t, y, z) d t+d h_{t}$, terminal time $\tau$ and terminal condition $X_{\tau}^{\prime}$. By the comparison theorem for BSDEs with default jump and generalized drivers provided in [10] (cf. Theorem 3 in [10]), we derive that $\mathcal{E}_{\sigma, \tau}^{\nu}\left(X_{\tau}^{\prime}\right) \leq X_{\sigma}^{\prime}$ a.s. It follows that $X^{\prime}$ is an $\mathcal{E}^{\nu}$-strong supermartingale for all $\nu \in \mathcal{V}$. By the minimality property of the process $X$ from Proposition 5.7, we derive that $X \leq X^{\prime}$. The proof is thus complete.

Proof of Theorem 4.15 (Infinitesimal characterization II): The result immediately follows from Theorem 4.13 together with Proposition B.5.

Proof of Theorem 4.6 (Optional $\mathcal{E}^{f}$-decomposition): The result follows from Theorem B.4 together with Remark 5.2. Moreover, since $Y$ is supposed to be right-continuous, we have $C=0$.

Proof of Remark 4.7: Since $Z^{\nu}=Z, K^{\nu}=K$ and $C=C^{\nu}=0$, the decomposition (B.3) from the proof of Proposition B.1 can be written:

$$
-d Y_{t}=f\left(t, Y_{t}, Z_{t}\right) d t-Z_{t} d W_{t}^{\nu}-K_{t} d M_{t}^{\nu}+d A_{t}^{\nu} .
$$


where $W^{\nu}$ and $M^{\nu}$ stand for $W^{Q^{\nu}}$ and $M^{Q^{\nu}}$ respectively. Hence, for each $\nu \in \mathcal{V}$, the processes $Z, K, A^{\nu}$ and $C=0$ correspond to the $\mathcal{E}_{Q^{\nu}}^{f}$-Mertens decomposition coefficients of the $Q^{\nu}$-square integrable strong $\mathcal{E}_{Q^{\nu}}^{f}$-supermartingale $Y$. We derive that $Z \in \cap_{\nu \in \mathcal{V}} \mathbb{H}_{Q^{\nu}}^{2}$, $K \in \cap_{\nu \in \mathcal{V}} \mathbb{H}_{Q^{\nu}, \lambda}^{2}$ and $A^{\nu} \in \cap_{\nu \in \mathcal{V}} S_{Q^{\nu}}^{2}$. By (B.4), we get $A \in \cap_{\nu \in \mathcal{V}} S_{Q^{\nu}}^{2}$. The result follows from Theorem B.4 and the equality (B.6) from the proof of Theorem B.4.

\section{A Some results on $\mathcal{E}^{g}$-supermartingale families and pro- cesses}

Let $g$ be a $\lambda$-admissible driver. We provide some results on $\mathcal{E}^{g}$-supermartingale families and $\mathcal{E}^{g}$-supermartingale processes, which are useful in the paper.

Lemma A.1. Let $(X(S), S \in \mathcal{T})$ be an $\mathcal{E}^{g}$-supermartingale family. Then, there exists a r.u.s.c. optional process $\left(X_{t}\right)$ such that $\mathbb{E}\left[\right.$ ess $\left.\sup _{S \in \mathcal{T}} X_{S}^{2}\right]<\infty$ which aggregates the family $(X(S), S \in \mathcal{T})$, that is, such that $X(S)=X_{S}$ a.s. for all $S \in \mathcal{T}$. Moreover, the process $\left(X_{t}\right)$ is a strong $\mathcal{E}^{g}$-supermartingale, that is, for all $S, S^{\prime} \in \mathcal{T}$ such that $S \geq S^{\prime}$ a.s., $\mathcal{E}_{S^{\prime}, S}^{g}\left(X_{S}\right) \leq$ $X_{S^{\prime}}$ a.s.

Proof. By Lemma 4.6 in [21], the $\mathcal{E}^{g}$-supermartingale family $(X(S), S \in \mathcal{T})$ is right-upper semicontinuous (along stopping times).

It follows from Theorem 4 in [8] that there exists an r.u.s.c. optional process $\left(X_{t}\right)$ which aggregates the family $(X(S), S \in \mathcal{T})$. The process $\left(X_{t}\right)$ is clearly a strong $\mathcal{E}^{g}$ supermartingale.

Remark A.2. Note that, as a consequence of the above lemma, we recover a result of [20] (Lemma 5.1 in [20]), namely, a strong $\mathcal{E}^{g}$-supermartingale is necessarily r.u.s.c.

We now recall the $\mathcal{E}^{g}$-Mertens decomposition of $\mathcal{E}^{g}$ - supermartingales proved in [20], and then provide some useful results.

Theorem A.3 ( $\mathcal{E}^{g}$-Mertens decomposition of $\mathcal{E}^{g}$-supermartingales). Let $\left(Y_{t}\right)$ be an optional process in $\mathbb{S}^{2}$. Then $\left(Y_{t}\right)$ is a $\mathcal{E}^{g}$-submartingale if and only if there exists a non decreasing right continuous and predictable processes $A$ in $\mathcal{A}^{2}$, a non decreasing adapted right continuous and purely discontinuous processes $C$ in $\mathbb{C}^{2}$ and $(Z, K) \in \mathbb{H}^{2} \times \mathbb{H}_{\nu}^{2}$ such that

$$
-d Y_{s}=g\left(s, Y_{s}, Z_{s}, K_{s}\right) d s-Z_{s} d W_{s}-K_{t} d M_{t}+d A_{s}+d C_{s^{-}} .
$$

Moreover, this decomposition is unique.

Remark A.4. Using the above decomposition, we deduce that a $\mathcal{E}^{g}$-supermartingale admits left and right limits.

Lemma A.5. If $\left(X_{t}\right)_{t \in[0, T]}$ be a strong $\mathcal{E}^{g}$-supermartingale, then the process of right-limits $\left(X_{t^{+}}\right)_{t \in[0, T]}$ (where, by convention, $X_{T^{+}}:=X_{T}$ ) is a strong $\mathcal{E}^{g}$-supermartingale. 
Proof. Since $\left(X_{t}\right)$ is a strong $\mathcal{E}^{g}$-supermartingale, $\left(X_{t}\right)$ has right limits (cf. Remark A.4). Let us show that the process $\left(X_{t^{+}}\right)$is a strong $\mathcal{E}^{g}$-supermartingale. Let $S, \theta$ be two stopping times belonging to $\mathcal{T}$ with $S \leq \theta$ a.s. There exist two nondecreasing sequences of stopping times $\left(S_{n}\right)$ and $\left(\theta_{n}\right)$ such that for each $n, S_{n}>S$ a.s. on $\{S<T\}$, and $\theta_{n}>\theta$ a.s. on $\{\theta<T\}$. Replacing if necessary $S_{n}$ by $S_{n} \wedge \theta_{n}$, we can suppose that for each $n, S_{n} \leq \theta_{n}$ a.s. Let $\nu \in \mathcal{V}$. Since the process $\left(X_{t}\right)$ is a strong $\mathcal{E}^{g}$-supermartingale, it follows that for each $n, \mathcal{E}_{S_{n}, \theta_{n}}^{g}\left(X_{\theta_{n}}\right) \leq X_{S_{n}}$ a.s. By the monotonicity property of $\mathcal{E}^{g}$, we derive that, for each $n$, $\mathcal{E}_{S, S_{n}}^{g}\left(\mathcal{E}_{S_{n}, \theta_{n}}^{g}\left(X_{\theta_{n}}\right)\right) \leq \mathcal{E}_{S, S_{n}}^{g}\left(X_{S_{n}}\right)$ a.s., which, by the consistency property of $\mathcal{E}^{g}$ implies

$$
\mathcal{E}_{S, \theta_{n}}^{g}\left(X_{\theta_{n}}\right) \leq \mathcal{E}_{S, S_{n}}^{g}\left(X_{S_{n}}\right) \text { a.s. }
$$

By letting $n$ tend to $\infty$ in the above inequality and by applying the continuity property (with respect to terminal time and terminal condition) of BSDEs with default (cf. [10]), we obtain

$$
\mathcal{E}_{S, \theta}^{g}\left(X_{\theta^{+}}\right) \leq \mathcal{E}_{S, S}^{g}\left(X_{S^{+}}\right)=X_{S^{+}} \text {a.s. }
$$

Hence, the process $\left(X_{t^{+}}\right)$is a strong $\mathcal{E}^{g}$-supermartingale.

Let $\mathcal{V}$ be a non-empty set. Let $\left(f^{\nu}, \nu \in \mathcal{V}\right)$ be a family of $\lambda$-admissible drivers satisfying Assumption 2.2.

Proposition A.6. Let $\eta$ be a given random variable belonging to $L^{2}\left(\mathcal{G}_{T}\right)$. Let $\left(X_{t}\right)_{t \in[0, T]}$ be an optional process such that $\left(X_{t}\right)$ is a strong $\mathcal{E}^{f^{\nu}}$-supermartingale for all $\nu \in \mathcal{V}$ and such that $X_{T} \geq \eta$ a.s. Assume moreover that $\left(X_{t}\right)$ is minimal, that is, $\left(X_{t}\right)$ is the smallest optional process satisfying these properties. Then, the process $\left(X_{t}\right)$ is right-continuous.

Proof. Since $\left(X_{t}\right)$ is a strong $\mathcal{E}^{f^{\nu}}$-supermartingale, it is r.u.s.c. (cf. Remark A.2) and has right limits (cf. Remark A.4). We thus have $X_{t^{+}} \leq X_{t}$, for all $t \in[0, T]$ a.s. Let us prove the converse inequality. Since $\left(X_{t}\right)$ is a strong $\mathcal{E}^{f^{\nu}}$-supermartingale for all $\nu \in \mathcal{V}$, it follows by Lemma A.5 that $\left(X_{t^{+}}\right)$is a strong $\mathcal{E}^{f^{\nu}}$-supermartingale for all $\nu \in \mathcal{V}$. We also note that $X_{T^{+}}=X_{T} \geq \eta$ a.s. Hence, using the minimality property of $\left(X_{t}\right)$, we derive that $X_{t} \leq X_{t^{+}}$, for all $t \in[0, T]$ a.s. We conclude that $X_{t^{+}}=X_{t}$, for all $t \in[0, T]$ a.s. The proof is thus complete.

Remark A.7. This property still holds in the case when the (terminal) constraint $X_{T} \geq \eta$ a.s. is replaced by the constraint $X_{t} \geq \xi_{t}$ for all $t \in[0, T]$ a.s., where $\left(\xi_{t}\right)$ is a given right lower-semicontinuous process belonging to $\mathbb{S}^{2} .^{12}$

\section{B Non-linear predictable and optional decompositions}

We provide a non-linear optional and a non-linear predictable decomposition for processes $\left(Y_{t}\right)$ which are strong $\mathcal{E}^{\nu}$-supermartingales for all $\nu \in \mathcal{V}$. In the present paper, we apply the

\footnotetext{
${ }^{12}$ The proof is analogous to the proof of the above Proposition A.6. The inequality $X_{T+} \geq \eta$ in the proof of Proposition A.6 is to be replaced by $X_{t+} \geq \xi_{t}$ for all $t \in[0, T]$ a.s., which holds due to the assumption of right lower-semicontinuity of $\left(\xi_{t}\right)$.
} 
decompositions of this section to the superhedging price processes of the European options. The decompositions prove useful also in our subsequent work on superhedging prices of American options (cf. [23]).

We denote by $\mathbb{C}^{2}$ the set of adapted non-decreasing RCLL purely discontinuous processes $\left(C_{t}\right)$ such that $C_{0-}=0$ and $E\left[C_{T}^{2}\right]<+\infty$.

Proposition B.1 (Predictable $\mathcal{E}^{f}$-decomposition). Let $\left(Y_{t}\right) \in \mathbb{S}^{2}$ be a strong $\mathcal{E}^{\nu}$-supermartingale for all $\nu \in \mathcal{V}$. There exists a unique process $(Z, K, A, C) \in \mathbb{H}^{2} \times \mathbb{H}_{\lambda}^{2} \times \mathcal{A}^{2} \times \mathbb{C}^{2}$ such that

$$
-d Y_{t}=f\left(t, Y_{t}, Z_{t}\right) d t-Z_{t} d W_{t}-K_{t} d M_{t}+d A_{t}+d C_{t-}
$$

and

$$
A .+\int_{0}^{\cdot}\left(K_{s}-\beta_{s} \sigma_{s}^{-1} Z_{s}\right) \lambda_{s} d s \in \mathcal{A}^{2} \quad \text { and } \quad\left(K_{t}-\beta_{t} \sigma_{t}^{-1} Z_{t}\right) \lambda_{t} \leq 0, t \in[0, T], d P \otimes d t-\text { a.e. }
$$

Remark B.2. Recall that by Remark 5 in [10], the condition $\left(K_{t}-\beta_{t} \sigma_{t}^{-1} Z_{t}\right) \lambda_{t} \leq 0$, $t \in$ $[0, T], d P \otimes d t-$ a.e. is equivalent to $K_{\vartheta}-\beta_{\vartheta} \sigma_{\vartheta}^{-1} Z_{\vartheta} \leq 0, P$-a.s.

Remark B.3. Note that excepting the default time $\vartheta$, the left-side jumps of $Y$ are predictable and correspond to the ones of the predictable non decreasing process $A$.

Proof. As $\left(Y_{t}\right)$ is a strong $\mathcal{E}^{0}$-supermartingale, by the $\mathcal{E}^{0}$-Mertens decomposition (see Theorem A.3 in Appendix), there exists a unique process $(Z, K, A, C)$ in $\mathbb{H}^{2} \times \mathbb{H}_{\lambda}^{2} \times \mathcal{A}^{2} \times \mathbb{C}^{2}$ such that equation (B.1) holds. Let $\nu \in \mathcal{V}$. Since $\left(Y_{t}\right)$ is a strong $\mathcal{E}^{\nu}$ - supermartingale, by the $\mathcal{E}^{\nu}$-Mertens decomposition (see A.3), there exists a unique process $\left(Z^{\nu}, K^{\nu}, A^{\nu}, C^{\nu}\right)$ in $\mathbb{H}^{2} \times \mathbb{H}_{\lambda}^{2} \times \mathcal{A}^{2} \times \mathbb{C}^{2}$ such that

$$
-d Y_{t}=\left(f\left(t, Y_{t}, Z_{t}^{\nu}\right)+\left(K_{t}^{\nu}-\beta_{t} \sigma_{t}^{-1} Z_{t}^{\nu}\right) \nu_{t} \lambda_{t}\right) d t-Z_{t}^{\nu} d W_{t}-K_{t}^{\nu} d M_{t}+d A_{t}^{\nu}+d C_{t-}^{\nu} .
$$

By applying the uniqueness of the canonical decomposition of a special optional semimartingale (cf. Lemma D.2 in the Appendix), together with the uniqueness of the representation of the martingale part as the sum of two stochastic integrals (with respect to $W$ and $M$ ), we have $Z_{t}=Z_{t}^{\nu} d P \otimes d t$-a.e. and $K_{t}=K_{t}^{\nu} d P \otimes d t$-a.e., $C_{t-}=C_{t-}^{\nu}$, for all $t$ a.s. and $f\left(t, Y_{t}, Z_{t}\right) d t+d A_{t}=f\left(t, Y_{t}, Z_{t}^{\nu}\right) d t+\left(K_{t}^{\nu}-\beta_{t} \sigma_{t}^{-1} Z_{t}^{\nu}\right) \nu_{t} \lambda_{t} d t+d A_{t}^{\nu}$ for all $t$ a.s.

Using the above equalities, we derive that

$$
d A_{t}^{\nu}=d A_{t}-\left(K_{t}-\beta_{t} \sigma_{t}^{-1} Z_{t}\right) \nu_{t} \lambda_{t} d t
$$

Let us show that this implies that $\left(K_{t}-\beta_{t} \sigma_{t}^{-1} Z_{t}\right) \lambda_{t} \leq 0, t \in[0, T], d P \otimes d t-$ a.e. Suppose by contradiction that there exists a predictable set $A \subset[0, T] \times \Omega$ such that $(d P \otimes d t)(A)>0$ and $\left(K_{t}-\beta_{t} \sigma_{t}^{-1} Z_{t}\right) \lambda_{t}>0, t \in[0, T], d P \otimes d t-$ a.e. on $A$. For each $n \in \mathbb{N}$, set $\nu_{t}^{n}:=n \mathbf{1}_{A}$. Note that $\left(\nu_{t}^{n}\right)$ is a bounded predictable process with $\nu_{t}^{n}>-1$. Hence, $\nu^{n} \in \mathcal{V}$. Using equality (B.4), we derive that for $n$ sufficiently large, we have $E\left[A_{T}^{\nu^{n}}\right]=E\left[A_{T}-n \int_{0}^{T}\left(K_{t}-\right.\right.$ $\left.\left.\beta_{t} \sigma_{t}^{-1} Z_{t}\right) \lambda_{t} \mathbf{1}_{A} d t\right]<0$. We thus get a contradiction with the non decreasing property of $A^{\nu^{n}}$. Hence, $\left(K_{t}-\beta_{t} \sigma_{t}^{-1} Z_{t}\right) \lambda_{t} \leq 0 d P \otimes d t$-a.s. 
Let us show that condition (B.4) implies that the process $A .+\int_{0}^{\cdot}\left(K_{s}-\beta_{s} \sigma_{s}^{-1} Z_{s}\right) \lambda_{s} d s$ is nondecreasing. Suppose by contradiction that there exist $B \in \mathcal{G}_{T}$ with $P(B)>0$, as well as $\varepsilon>0$ and $(t, s) \in[0, T]^{2}$ with $t<s$, such that $\int_{t}^{s}\left(d A_{r}+\left(K_{r}-\beta_{r} \sigma_{r}^{-1} Z_{r}\right) \lambda_{r} d r\right) \leq-\varepsilon$ a.s. on $B$. For each $n \in \mathbb{N}^{*}$, set $\nu^{n}:=-1+\frac{1}{n}$. Note that $\nu^{n} \in \mathcal{V}$. From (B.4), we derive that $\int_{t}^{s}\left(d A_{r}+\left(K_{r}-\beta_{r} \sigma_{r}^{-1} Z_{r}\right)\left(-1+\frac{1}{n}\right) \lambda_{r} d r\right) \geq 0$ a.s. We thus get that for all $n \in \mathbb{N}^{*}$,

$$
-\varepsilon \geq \int_{t}^{s}\left(d A_{r}+\left(K_{r}-\beta_{r} \sigma_{r}^{-1} Z_{r}\right) \lambda_{r} d r\right) \geq \frac{1}{n} \int_{t}^{s}\left(K_{r}-\beta_{r} \sigma_{r}^{-1} Z_{r}\right) \lambda_{r} d r \text { a.s. on B. }
$$

By letting $n$ tend to $+\infty$ in this inequality, we obtain a contradiction. Hence, the process $A .+\int_{0}^{\cdot}\left(K_{s}-\beta_{s} \sigma_{s}^{-1} Z_{s}\right) \lambda_{s} d s$ is nondecreasing.

Moreover, the uniqueness of the decomposition follows by Lemma D.2.

Theorem B.4 (Optional $\mathcal{E}^{f}$-decomposition). Let $\left(Y_{t}\right)$ be an optional process belonging to $\mathbb{S}^{2}$. Suppose that it is an $\mathcal{E}^{\nu}$-strong supermartingale for each $\nu \in \mathcal{V}$.

Then, there exists a unique $Z \in \mathbb{H}^{2}$, a unique $C \in \mathbb{C}^{2}$ and a unique nondecreasing optional $R C L L$ process $h$, with $h_{0}=0$ and $E\left[h_{T}^{2}\right]<\infty$ such that

$$
-d Y_{t}=f\left(t, Y_{t}, Z_{t}\right) d t-Z_{t} \sigma_{t}^{-1}\left(\sigma_{t} d W_{t}+\beta_{t} d M_{t}\right)+d C_{t^{-}}+d h_{t}
$$

Proof. By Proposition B.1, there exists $(Z, K, A, C) \in \mathbb{H}^{2} \times \mathbb{H}_{\lambda}^{2} \times \mathcal{A}^{2} \times \mathbb{C}^{2}$ such that (B.1) and (B.2) hold. Set $h_{t}:=A_{t}-\int_{0}^{t}\left(K_{s}-\beta_{s} \sigma_{s}^{-1} Z_{s}\right) d M_{s}$. Since $d M_{t}=d N_{t}-\lambda_{t} d t$, we have

$$
h_{t}=A_{t}+\int_{0}^{t}\left(K_{s}-\beta_{s} \sigma_{s}^{-1} Z_{s}\right) \lambda_{s} d s-\int_{0}^{t}\left(K_{s}-\beta_{s} \sigma_{s}^{-1} Z_{s}\right) d N_{s}
$$

Now, by property (B.2), the process $A .+\int_{0}^{\cdot}\left(K_{s}-\beta_{s} \sigma_{s}^{-1} Z_{s}\right) \lambda_{s} d s$ is non decreasing.

Moroever, the process $\int_{0}^{\cdot}\left(K_{s}-\beta_{s} \sigma_{s}^{-1} Z_{s}\right) d N_{s}$ is a purely discontinuous process which admits a unique jump, given by $K_{\vartheta}-\beta_{\vartheta} \sigma_{\vartheta}^{-1} Z_{\vartheta}$ (at time $\vartheta$ ). Now by Remark B.2, we have $K_{\vartheta}-$ $\beta_{\vartheta} \sigma_{\vartheta}^{-1} Z_{\vartheta} \leq 0$ a.s. We thus derive that the process $\int_{0}^{\cdot}\left(K_{s}-\beta_{s} \sigma_{s}^{-1} Z_{s}\right) d N_{s}$ is non increasing. Hence, by the equality (B.6), we derive that the process $\left(h_{t}\right)$ is non decreasing. Using (B.1), we thus get the equation (B.5).

It remains to show the uniqueness of the processes $Z, C$, and $h$ in (B.5). To show this, we first show that if $Y$ is decomposable as in (B.5), then the process $Y^{\prime}$ defined by $Y_{t}^{\prime}=Y_{t}-\Delta Y_{\vartheta} \mathbb{I}_{t \geq \vartheta}$ is a special optional semimartingale (cf. Appendix). By equation (B.5), we have

$$
\Delta Y_{\vartheta}=Z_{\vartheta} \sigma_{\vartheta}^{-1} \beta_{\vartheta}-\Delta h_{\vartheta}
$$

Subtracting $\Delta Y_{\vartheta} \mathbb{I}_{t \geq \vartheta}$ on both sides of the equation (B.5), we get

$$
Y_{t}-\Delta Y_{\vartheta} \mathbb{I}_{t \geq \vartheta}=Y_{0}-\int_{0}^{t} f\left(s, Y_{s}, Z_{s}\right) d s+\int_{0}^{t} Z_{s} \sigma_{s}^{-1}\left(\sigma_{s} d W_{s}+\beta_{s} d M_{s}\right)-C_{t^{-}}-h_{t}-\Delta Y_{\vartheta} \mathbb{I}_{t \geq \vartheta}
$$


Using this and the expression (B.7) for $\Delta Y_{\vartheta}$, we get

$Y_{t}-\Delta Y_{\vartheta} \mathbb{I}_{t \geq \vartheta}=Y_{0}-\int_{0}^{t} f\left(s, Y_{s}, Z_{s}\right) d s+\int_{0}^{t} Z_{s} \sigma_{s}^{-1}\left(\sigma_{s} d W_{s}+\beta_{s} d M_{s}\right)-C_{t^{-}}-h_{t}-Z_{\vartheta} \sigma_{\vartheta}^{-1} \beta_{\vartheta} \mathbb{I}_{t \geq \vartheta}+\Delta h_{\vartheta} \mathbb{I}_{t \geq \vartheta}$

We set $B_{t}:=h_{t}-\Delta h_{\vartheta} \mathbb{I}_{t>\vartheta}$. By Lemma D.3, the process $B$ is a (predictable) process in $\mathcal{A}^{2}$. Recall that we have also set $Y_{t}^{\prime}=Y_{t}-\Delta Y_{\vartheta} \mathbb{I}_{t \geq \vartheta}$. With this notation, equation (B.9) becomes

$$
Y_{t}^{\prime}=Y_{0}^{\prime}-\int_{0}^{t} f\left(s, Y_{s}, Z_{s}\right) d s+\int_{0}^{t} Z_{s} \sigma_{s}^{-1}\left(\sigma_{s} d W_{s}+\beta_{s} d M_{s}\right)-C_{t^{-}}-B_{t}-Z_{\vartheta} \sigma_{\vartheta}^{-1} \beta_{\vartheta} \mathbb{I}_{t \geq \vartheta} .
$$

Since $d M_{t}=d N_{t}-\lambda_{t} d t$, we get

$$
Y_{t}^{\prime}=Y_{0}^{\prime}-\int_{0}^{t} f\left(s, Y_{s}, Z_{s}\right) d s+\int_{0}^{t} Z_{s} d W_{s}-C_{t^{-}}-B_{t}-\int_{0}^{t} Z_{s} \sigma_{s}^{-1} \beta_{s} \lambda_{s} d s .
$$

We conclude that $Y^{\prime}$ is a special optional semimartingale.

Let now $\tilde{Z}, \tilde{C}$, and $\tilde{h}$ be such that $\tilde{Z} \in \mathbb{H}^{2}, \tilde{C} \in \mathbb{C}^{2}$ and $\tilde{h}$ is a nondecreasing optional RCLL process with $\tilde{h}_{0}=0$ and $E\left[\tilde{h}_{T}^{2}\right]<\infty$, and such that the decomposition (B.5) holds with $\tilde{Z}$, $\tilde{C}$, and $\tilde{h}$ (in place of $Z, C, h$ ). We show that $\tilde{Z}=Z$ in $\mathbb{H}^{2}, \tilde{C}_{t}=C_{t}$, for all $t$ a.s. and $\tilde{h}_{t}=h_{t}$, for all $t$ a.s. By the same reasoning as above, we have that (B.11) holds also with $Z$, $C$, and $B$ replaced by $\tilde{Z}, \tilde{C}$, and $\tilde{B}$, where $\tilde{B}$ is defined by $\tilde{B}_{t}:=\tilde{h}_{t}-\Delta \tilde{h}_{\vartheta} \mathbb{I}_{t \geq \vartheta}$. We note that, due to (B.7), $\Delta \tilde{h}_{\vartheta}=\Delta h_{\vartheta}$. Hence, showing the equality $\tilde{h}_{t}=h_{t}$, for all $t$ a.s. is equivalent to showing that $\tilde{B}_{t}=B_{t}$, for all $t$ a.s.

Now, as $Y^{\prime}$ is a special optional semimartingale admitting the decomposition (B.11) with $Z$, $C$, and $B$, on one hand, and with $\tilde{Z}, \tilde{C}$, and $\tilde{B}$, on the other hand, we have, by the uniqueness of the special optional semimartingale decomposition (cf. Lemma D.1 in the Appendix), that $C=\tilde{C}, f\left(t, Y_{t}, Z_{t}\right) d t+d B_{t}+Z_{t} \sigma_{t}^{-1} \beta_{t} \lambda_{t} d t=f\left(t, Y_{t}, \tilde{Z}_{t}\right) d t+d \tilde{B}_{t}+\tilde{Z}_{t} \sigma_{t}^{-1} \beta_{t} \lambda_{t} d t$, and $Z_{t} d W_{t}=$ $\tilde{Z}_{t} d W_{t}$. From the last equality, using the uniqueness of the martingale representation, we get $Z=\tilde{Z}$ in $\mathbb{H}^{2}$. This, together with the second equality, gives the equality of $B$ and $\tilde{B}$. The proof is thus complete.

Proposition B.5. Let $\left(Y_{t}\right) \in \mathbb{S}^{2}$. The process $\left(Y_{t}\right)$ admits an optional decomposition of the form (B.5) if and only if it admits a decomposition of the form (B.1) with the conditions (B.2).

Proof. By the same arguments as those used in the proof of Theorem B.4, we derive that if $\left(Y_{t}\right)$ admits a decomposition of the form (B.1) with conditions (B.2), then it admits an optional decomposition of the form (B.5).

It remains to show the converse. Suppose that there exists $(Z, C) \in \mathbb{H}^{2} \times \mathbb{C}^{2}$ and a nondecreasing optional RCLL process $h$, with $h_{0}=0$ and $E\left[h_{T}^{2}\right]<\infty$ such that the equation (B.5) holds. By Lemma D.3, $h$ has the following decomposition $h_{t}=B_{t}+\int_{0}^{t} \psi_{s} d N_{s}$, where 
$B$ is a (predictable ) process in $\mathcal{A}^{2}$ and $\psi \in \mathbb{H}_{\lambda}^{2}$ with $\psi_{t} \lambda_{t} \geq 0 d P \otimes d t$-a.s. Let $\left(A_{t}\right)$ be the process defined for all $t \in[0, T]$ by

$$
A_{t}:=B_{t}+\int_{0}^{t} \psi_{s} \lambda_{s} d s .
$$

We have $A \in \mathcal{A}^{2}$. Let $\left(K_{t}\right)$ be the process defined for all $t \in[0, T]$ by

$$
K_{t}:=\beta_{t} \sigma_{t}^{-1} Z_{t}-\psi_{t}
$$

Note that $K \in \mathbb{H}_{\lambda}^{2}$. Now, since $\psi_{t} \lambda_{t} \geq 0 d P \otimes d t$-a.s., we have $\left(K_{t}-\beta_{t} \sigma_{t}^{-1} Z_{t}\right) \lambda_{t} \leq 0$ $d P \otimes d t$-a.s. Moreover, by (B.12) and (B.13), we have $B_{t}=A_{t}+\int_{0}^{t}\left(K_{s}-\beta_{s} \sigma_{s}^{-1} Z_{s}\right) \lambda_{s} d s$. Since $B \in \mathcal{A}^{2}$, we derive that the conditions (B.2) hold.

Moreover, since $N_{t}=M_{t}+\int_{0}^{t} \lambda_{s} d s$, by (B.12), we get $h_{t}=B_{t}+\int_{0}^{t} \psi_{s} d N_{s}=A_{t}+\int_{0}^{t} \psi_{s} d M_{s}$ a.s. Hence, using (B.5) and (B.13), we derive that the process $(Z, K, A, C)$ satisfies the equation (B.1). The proof is thus complete.

\section{A result on BSDEs with a non positive jump at the default time $\vartheta$}

Let $\mathcal{V}^{\prime}$ be the set of bounded predictable processes $\nu$ such that $\nu_{t} \geq 0 d P \otimes d t$-a.e.

Let $g$ be a $\lambda$-admissible driver and let $\left(\delta_{t}\right)$ be a bounded predictable process.

Let $\eta$ be a $\mathcal{G}_{T}$-measurable random variable satisfying Assumption (4.1). For each $\nu \in \mathcal{V}^{\prime}$, we define

$$
g^{\nu}(\omega, t, y, z, k):=g(\omega, t, y, z, k)+\nu_{t}(\omega) \lambda_{t}(\omega)\left(k-\delta_{t}(\omega) z\right)
$$

Note that $g^{\nu}$ is a $\lambda$-admissible driver. Let $\eta$ be a For each $S \in \mathcal{T}$, the value $X(S)$ at time $S$ is defined by

$$
X(S):=e s s \sup _{\nu \in \mathcal{V}^{\prime}} \mathcal{E}_{S, T}^{\nu}(\eta)
$$

where $\mathcal{E}^{\nu}=\mathcal{E}^{g^{\nu}}$. Note that $X_{T}=\eta$ a.s. By Proposition A.6 and similar arguments as in the previous case (cf. the proof of Proposition 5.7), there exists an RCLL process $\left(X_{t}\right) \in S^{2}$ which aggregates the value family $(X(S))$, which is a strong $\mathcal{E}^{\nu}$-supermartingale for all $\nu \in \mathcal{V}^{\prime}$ and $X_{T} \geq \eta$ a.s. Moreover, the process $\left(X_{t}\right)$ is the smallest process in $S^{2}$ satisfying these properties.

Now, by similar arguments as those used in the proof of Proposition B.1, it can be shown that

Proposition C.1. Let $\left(Y_{t}\right) \in \mathbb{S}^{2}$. If the process $\left(Y_{t}\right)$ is a strong $\mathcal{E}^{\nu}$-supermartingale for all $\nu \in \mathcal{V}^{\prime}$, then there exists a unique process $(Z, K, A, C) \in \mathbb{H}^{2} \times \mathbb{H}_{\lambda}^{2} \times \mathcal{A}^{2} \times \mathbb{C}^{2}$ such that

$$
-d Y_{t}=g\left(t, Y_{t}, Z_{t}, K_{t}\right) d t-Z_{t} d W_{t}-K_{t} d M_{t}+d A_{t}+d C_{t-}
$$


and

$$
\left(K_{t}-\delta_{t} Z_{t}\right) \lambda_{t} \leq 0, t \in[0, T], d P \otimes d t-\text { a.e. }
$$

Moreover, the converse statement holds.

Note that when $\delta=0$, the constraint (C.3) means that the jump of the process $\left(Y_{t}\right)$ at the default time $\vartheta$ is non positive.

Remark C.2. The constraint (C.3) is equivalent to $K_{\vartheta} \leq \delta_{\vartheta} Z_{\vartheta}$ a.s. Note that this constraint corresponds to the second constraint from (B.2). There is here only one constraint (C.3) while in the previous case, we had two constraints (see (B.2)). This comes from the fact that here $\mathcal{V}^{\prime}$ is the set of bounded predictable processes $\nu$ with $\nu_{t} \geq 0 d P \otimes d t$-a.e., while in the (previous) case of $\mathcal{V}$, we had $\nu_{t}>-1 d P \otimes d t$-a.e.

By similar arguments as those used in the proof of Theorem 4.15, it can be shown that the value process $\left(X_{t}\right)$ is a supersolution of the constrained reflected BSDE from Definition 4.14 with $f$ replaced by $g$ and the constraints (B.2) replaced by the constraint (C.3). We thus have the following result.

Proposition C.3. Let $\left(X_{t}\right) \in S^{2}$ be the RCLL process which aggregates the value family $(X(S))$ defined by (C.1). There exists a unique process $(Z, K, A) \in \mathbb{H}^{2} \times \mathbb{H}_{\lambda}^{2} \times \mathcal{A}^{2}$ such that

$$
\begin{aligned}
& -d X_{t}=g\left(t, X_{t}, Z_{t}, K_{t}\right) d t+d A_{t}-Z_{t} d W_{t}-K_{t} d M_{t} ; X_{T}=\eta ; \\
& \left(K_{t}-\delta_{t} Z_{t}\right) \lambda_{t} \leq 0, t \in[0, T], d P \otimes d t-\text { a.e. }
\end{aligned}
$$

In other words, the value process $\left(X_{t}\right)$ is a supersolution of the above constrained BSDE. Moreover, it is the minimal one, that is, if $\left(X_{t}^{\prime}\right)$ is another supersolution, then $X_{t}^{\prime} \geq X_{t}$ for all $t \in[0, T]$ a.s.

Note that when $\delta=0$, our result gives the existence of a minimal supersolution of the BSDE with driver $g$, terminal condition $\eta$ and with non positive jumps, which corresponds to a result shown in [4] by using a penalization approach. Moreover, our result provides a dual representation (with non linear expectation) of this minimal supersolution.

\section{Three useful lemmas}

Lemma D.1. Let $g$ be a $\lambda$-admissible driver. Let $\left(A_{t}\right)$ be a RCLL predictable process with square integrable total variation and $A_{0}=0$. Suppose $X$ is the first component of the solution of both the BSDE with generalized driver $g(\cdot, y, z, k) d t+d A_{t}$ and the BSDE with driver $g$ (with the same terminal time $T$ and the same terminal condition $\eta \in L^{2}\left(\mathcal{G}_{T}\right)$ ). We then have $A_{t}=0$ for all $t \in[0, T]$ a.s.

Sketch of the proof: By assumption, there exists a unique process $(Z, K)$ in $\mathbb{H}^{2} \times \mathbb{H}_{\lambda}^{2}$ such that $(X, Z, K)$ satisfies (2.2). Also, there exists ${ }^{13}$ a unique $\left(Z^{\prime}, K^{\prime}\right)$ in $\mathbb{H}^{2} \times \mathbb{H}_{\lambda}^{2}$ such that

\footnotetext{
${ }^{13} \mathrm{cf}$. [10] for the existence and the uniqueness of the solution of the BSDE with default and generalized driver.
} 
$\left(X, Z^{\prime}, K^{\prime}\right)$ satisfies $-d X_{t}=g\left(t, X_{t}, Z_{t}^{\prime}, K_{t}^{\prime}\right) d t+d A_{t}-Z_{t}^{\prime} d W_{t}-K_{t}^{\prime} d M_{t}$. By the uniqueness of the decomposition of a special semi-martingale together with the uniqueness in the martingale representation, we derive that $Z=Z^{\prime}$ in $\mathbb{H}^{2}$ and $K=K^{\prime}$ in $\mathbb{H}_{\lambda}^{2}$, and $d A_{t}=0$. The proof is thus complete.

Lemma D.2. (Uniqueness of the canonical decomposition of a special optional semimartingale) Let $X$ be an optional semimartingale with decomposition ${ }^{14}$

$$
X_{t}=X_{0}+m_{t}-a_{t}-b_{t}, \text { for all } t \in[0, T] \text { a.s. }
$$

with $\left(m_{t}\right)$ a (right-continuous) local martingale, $\left(a_{t}\right)$ a predictable right-continuous process of finite variation, such that $a_{0}=0,\left(b_{t}\right)$ a predictable left-continuous process of finite variation, purely discontinuous and such that $b_{0-}=0$. Then, the decomposition (D.1) is unique and will be called the canonical decomposition of a special optional semimartingale.

Proof. Let $X_{t}=X_{0}+m_{t}^{\prime}-a_{t}^{\prime}-b_{t}^{\prime}$, for all $t \in[0, T]$ a.s., be (another) decomposition with $\left(m_{t}^{\prime}\right),\left(a_{t}^{\prime}\right)$ and $\left(b_{t}^{\prime}\right)$ as in the lemma. From this decomposition, it follows that $X_{t+}-X_{t}=$ $-\left(b_{t+}^{\prime}-b_{t}^{\prime}\right)$ for all $t$ a.s. From (D.1), it follows that $X_{t+}-X_{t}=-\left(b_{t+}-b_{t}\right)$ for all $t$ a.s. Hence, $b_{t+}^{\prime}-b_{t}^{\prime}=b_{t+}-b_{t}$ for all $t$ a.s. As $b$ and $b^{\prime}$ are purely discontinuous with the same initial value, we get $b_{t}^{\prime}=b_{t}$, for all $t$ a.s. and the uniqueness of $b$ is proven. We now note that $\left(X_{t}+b_{t}\right)_{t}$ is a special right-continuous semimartingale (this follows from (D.1)). Hence, by Theorem 30, Chapter III in [34] the processes $\left(m_{t}\right)$ and $\left(a_{t}\right)$ are unique.

Lemma D.3. Let $h$ be a nondecreasing optional $R C L L$ process $h$, with $h_{0}=0$ and $E\left[h_{T}^{2}\right]<$ $\infty$. Then, $h$ has at most one totally inaccessible jump and this jump is at $\vartheta$. All the other jumps of $h$ are predictable. Moreover, $h$ can be uniquely decomposed as follows:

$$
h_{t}=B_{t}+\Delta h_{\vartheta} \mathbb{I}_{t \geq \vartheta}=B_{t}+\int_{0}^{t} \psi_{s} d N_{s},
$$

where $B$ is a (predictable) process in $\mathcal{A}^{2}$ and $\psi$ is a process in $\mathbb{H}_{\lambda}^{2}$ such that $\psi_{\theta} \geq 0$ a.s. on $\{\theta \leq T\}$.

Proof. As $h$ is a square-integrable nondecreasing optional RCLL process, $h$ is a squareintegrable RCLL submartingale. So, by the classical Doob-Meyer decomposition, $h$ can be uniquely decomposed as $h_{t}=a_{t}+m_{t}$, with $\left(a_{t}\right)$ a (predictable) process in $\mathcal{A}^{2}$ and $\left(m_{t}\right)$ a square-integrable martingale such that $m_{0}=0$. Now, by the martingale representation of $\mathbb{G}$-martingales and as $d M_{s}=d N_{s}-\lambda_{s} d s$, we get $m_{t}=\int_{0}^{t} \varphi_{s} d W_{s}-\int_{0}^{t} \psi_{s} \lambda_{s} d s+\int_{0}^{t} \psi_{s} d N_{s}$, where $\varphi \in \mathbb{H}^{2}$ and $\psi \in \mathbb{H}_{\lambda}^{2}$. Hence, $h_{t}=a_{t}+m_{t}=B_{t}+\int_{0}^{t} \psi_{s} d N_{s}=B_{t}+\psi_{\vartheta} \mathbb{I}_{t \geq \vartheta}$, where we have set $B_{t}:=a_{t}+\int_{0}^{t} \varphi_{s} d W_{s}-\int_{0}^{t} \psi_{s} \lambda_{s} d s$. The process $\left(B_{t}\right)$ is clearly predictable (as the sum of three predictable processes). The equality $h_{t}=B_{t}+\psi_{\vartheta} \mathbb{I}_{t \geq \vartheta}$, together with the predictability of $B$ and the non-decreasingness of $h$, implies that $\Delta h_{\vartheta}=\psi_{\vartheta} \geq 0$ a.s. on $\{\theta \leq T\}$ and that $B$ is non-decreasing. The proof is thus complete.

\footnotetext{
${ }^{14}$ An optional semimartingale with a decomposition of this from (with $\left(a_{t}\right)$ and $\left(b_{t}\right)$ predictable processes) can be seen as a generalisation of the notion of special semimartingale from the right-continuous to the general case.
} 


\section{References}

[1] Bank, P. and Kramkov D. (2015): A model for a large investor trading at market indifference prices. II: Continuous-time case, The Annals of Applied Probability No. 5, $2708-2742$

[2] Bouchard B., D. Possamaï, and X. Tan (2016): A general Doob-Meyer-Mertens decomposition for $g$-supermartingale system, Electronic Journal of Probability 21, paper no. 36,21 pages.

[3] Brigo, D., Franciscello, M., and Pallavicini: Analysis of nonlinear valuation equations under credit and funding effects. In Innovations in Derivative Markets, Springer Proceedings in Mathematics and Statistics, Vol. 165, Glau, K., Grbac, Z., Scherer, M., and Zagst, R. (Eds.), Springer, Heidelberg, 2016, 37-52.

[4] Kharroubi I., Ma J., Pham H., and J. Zhang (2010) Backward SDEs with constrained jumps and quasi-variational inequalities, Annals of Probability, 38, 794-840.

[5] Crepey, S, Bilateral Counterparty Risk under Funding Constraints- Part 1 CVA. Pricing. Mathematical Finance 25 (1), 1-22, 2015. 113, 2015.

[6] Cvitanić J. and Karatzas, I., Hedging Contingent Claims with Constrained Portfolios, Annals of Applied Prob. 1993.

[7] Cvitanić J. and Ma, J., Hedging options for a large investor and forward-backward SDEs, Annals of applied Prob. 1996. 6, n.2 370-398.

[8] Dellacherie C. and E. Lenglart (1981): Sur des problèmes de régularisation, de recollement et d'interpolation en théorie des processus, Sém. de Proba. XVI, lect. notes in Mathematics, 920, 298-313, Springer-Verlag.

[9] Dellacherie, C. and Meyer, P.-A. (1975). Probabilités et Potentiel, Chap. I-IV. Nouvelle édition. Hermann. MR0488194

[10] Dumitrescu, R., Grigorova, M., Quenez M.C., Sulem A. (2018), BSDEs with default jump, in Computation and Combinatorics in Dynamics, Stochastics and Control Abel Symposium, August 2016, E. Celledoni et al. (Eds) vol 13. Springer, p. 233-263, https://doi.org/10.1007/978-3-030-01593-0_9, available at https://hal.inria.fr/hal-01799335.

[11] Dumitrescu, R., Quenez M.C., and A. Sulem, Game options in an imperfect market with default, SIAM Journal on Financial Mathematics, Vol 8, pp 532-559, 2017.

[12] Dumitrescu R., Quenez M-C., and Sulem, A. (2018) American options in an imperfect complete market with default, ESAIM Proceedings \&3 Surveys, Vol 64, pp 93-110. 
[13] El Karoui N., Peng S. and M.C. Quenez (1997), "Backward Stochastic Differential Equations in Finance", Mathematical Finance, 7, 1 (January 1997), 1-71.

[14] El Karoui N., Peng S. and M.C. Quenez (2001), "A dynamic maximum principle for the optimization of recursive utilities under constraints", Vol.11, n.3, 664-693 Annals of Applied Probability.

[15] N. El Karoui and M.-C. Quenez (1991), Programmation dynamique et évaluation des actifs contingents en marché incomplet. Comptes rendus de l'Académie des sciences. Série 1, Mathématique, 313 (12) 851-854, 1991.

[16] El Karoui N. and M.C. Quenez (1995), Dynamic programming and pricing of contingent claims in an incomplete market, SIAM J. Control and Optimization, 33, 29-66.

[17] El Karoui N. and M.C. Quenez (1996), "Non-linear Pricing Theory and Backward Stochastic Differential Equations", Lectures Notes in Mathematics 1656, Bressanone, 1996, Editor:W.J.Runggaldier, collection Springer,1997.

[18] Föllmer H. and Kabanov Y.M., Optional decomposition and Lagrange multipliers, Finance and Stochastics, 2,1,pp. 66-81,1997.

[19] Gal'chouk L. I. (1981) : Optional martingales, Math. USSR Sbornik 40(4), 435-468.

[20] Grigorova, M., Imkeller, P., Offen, E., Ouknine, Y., and Quenez, M.-C. (2017): Reflected BSDEs when the obstacle is not right-continuous and optimal stopping, the Annals of Applied Probability, 27(5), 3153-3188.

[21] Grigorova, M., Imkeller, P., Ouknine, Y., and Quenez, M.-C. (2016): Optimal stopping with $f$-expectations: the irregular case, arXiv:1611.09179, submitted.

[22] Grigorova M., P. Imkeller, Ouknine, Y., and Quenez, M.-C. (2018): Doubly Reflected BSDEs and $\mathcal{E}^{f}$-Dynkin games: beyond the right-continuous case, Electron. J. Probab. 23, paper 122, 38 pages.

[23] Grigorova, M., Quenez, M.C., and Sulem, A. (2018): American options in a non-linear incomplete market model with default, preprint.

[24] Harrison J.M. and Kreps D.M., Martingales and arbitrage in multiperiod securities markets, J.Econom. Theory, 20 (1979), pp. 381-408.

[25] Jeanblanc M. and Song S., Martingale representation theorem in progressively enlarged filtrations Stochastic Processes and their Applic., 125, 4242-4271, 2015.

[26] Jeanblanc, M., Yor M., and Chesney M. (2009): Mathematical methods for financial markets, Springer Finance.

[27] Karatzas, I. and Kou, S. (1996): On the pricing of contingent claims under constraints, Ann. Appl. Probab. 6(2), 321-369. 
[28] Karatzas, I. and Shreve, S.E., Methods of Mathematical Finance. Applications of Mathematics (New York) 39. Springer, New York, 1998.

[29] Korn R., Contingent claim valuation in a market with different interest rates, Mathematical Methods of Operation Research (1995) 42, 255-274.

[30] Kusuoka, S., A remark on default risk models, Adv. Math. Econ., 1, 69-82, 1999.

[31] Kramkov D., Optional decomposition of supermartingales and hedging contingent claims in incomplete security markets, Probab. Theory Relat. Fields, 105, 459-479, 1996.

[32] Kramkov, D.; Schachermayer, W. The asymptotic elasticity of utility functions and optimal investment in incomplete markets. Ann. Appl. Probab. 9 (1999), no. 3, 904950.

[33] Peng, S. (2004), Nonlinear expectations, nonlinear evaluations and risk measures, 165253, Lecture Notes in Math., Springer, Berlin.

[34] Protter, P. (2004) Stochastic integration and differential equations, second edition, Springer-Verlag Berlin Heidelberg New York.

[35] Schachermayer, W. Optimal investment in incomplete financial markets, in Stochastic Methods in Finance: Lectures given at the C.I.M.E.-E.M.S. Summer School,held in Bressanone/Brixen, Italy, July 6-12, 2003, Springer, eds M. Fritelli and W. Runggaldier. 